\title{
Calabi-Yau threefolds in $\mathbb{P}^{6}$
}

\author{
Grzegorz Kapustka • Michał Kapustka
}

Received: 7 October 2013 / Accepted: 14 January 2015 / Published online: 31 January 2015

(C) The Author(s) 2015. This article is published with open access at Springerlink.com

\begin{abstract}
We study the geometry of 3-codimensional smooth subvarieties of the complex projective space. In particular, we classify all quasi-Buchsbaum Calabi-Yau threefolds in projective 6-space. Moreover, we prove that this classification includes all Calabi-Yau threefolds contained in a possibly singular 5-dimensional quadric as well as all Calabi-Yau threefolds of degree at most 14 in $\mathbb{P}^{6}$.
\end{abstract}

Keywords Calabi-Yau threefolds · Pfaffian varieties · Canonical surfaces

Mathematics Subject Classification Primary: 14J32

\section{Introduction}

It is conjectured that, when $2 n \geq N$, there is a finite number of smooth families of smooth $n$-dimensional subvarieties of $\mathbb{P}^{N}$ that are not of general type. This conjecture was inspired by [15] where the statement was formulated and proven in the case of surfaces in $\mathbb{P}^{4}$. In [9], the conjecture was proven in the case of threefolds in $\mathbb{P}^{5}$. Moreover, Schneider in [33] proved that the statement is true when $2 n \geq N+2$. In this context, it is a natural problem to classify

\footnotetext{
G. Kapustka · M. Kapustka (凶)

Institut für Mathematik, Mathematisch-naturwissenschaftliche Fakultät, Universität Zürich, Winterthurerstrasse 190, 8057 Zurich, Switzerland e-mail: michal.kapustka@math.uzh.ch; michal.kapustka@uj.edu.pl

G. Kapustka

e-mail: grzegorz.kapustka@uj.edu.pl

G. Kapustka $\cdot$ M. Kapustka

Department of Mathematics and Informatics, Jagiellonian University, Łojasiewicza 6, 30-348 Kraków, Poland

G. Kapustka · M. Kapustka

Institute of Mathematics of the Polish Academy of Sciences,

ul. Śniadeckich 8, P.O. Box 21, 00-956 Warszawa, Poland
} 
families of smooth $n$-folds of small degree in $\mathbb{P}^{N}$ for chosen $n, N \in \mathbb{N}$ satisfying $2 n \geq N$. In the case of codimension 2 subvarieties, this problem was addressed by many authors (see $[3,6,11,17])$.

The next step is to study codimension 3 subvarieties in $\mathbb{P}^{6}$. In this case, standard tools such as the Barth-Lefschetz theorem do not apply. However, some general structure theorems were recently developed. We say that a submanifolds $X \subset \mathbb{P}^{n}$ is subcanonical when $\omega_{X}=\mathcal{O}_{X}(k)$ for some $k \in \mathbb{Z}$. A codimension 3 submanifolds $X$ is called Pfaffian if it is the first nonzero degeneracy locus of a skew-symmetric morphism of vector bundles of odd rank $E^{*}(-t) \stackrel{\varphi}{\rightarrow} E$ where $t \in \mathbb{Z}$. In this case, $X$ is given locally by the vanishing of $2 u \times 2 u$ Pfaffians of an alternating map $\varphi$ from the vector bundle $E$ of odd rank $2 u+1$ to its twisted dual. More precisely, if $X$ is Pfaffian, then we have:

$$
0 \rightarrow \mathcal{O}_{\mathbb{P}^{n}}(-2 s-t) \rightarrow E^{*}(-s-t) \stackrel{\varphi}{\rightarrow} E(-s) \rightarrow \mathcal{I}_{X} \rightarrow 0
$$

where $s=c_{1}(E)+u t$. Moreover, from [29, Sect.3], we have in this case

$$
\omega_{X}=\mathcal{O}_{X}(t+2 s-n-1) .
$$

Since the choice of an alternating map $\varphi$ is equivalent to the choice of a section $\sigma \in$ $H^{0}\left(\bigwedge^{2} E(t)\right)$, we will use the notation $\operatorname{Pf}(\sigma)$ for the variety described by the Pfaffians of the map corresponding to $\sigma$.

Answering a question of Okonek (see [29]), Walter, in [37], proved that if $n$ is not divisible by 4 then a locally Gorenstein codimension 3 submanifold of $\mathbb{P}^{n+3}$ is Pfaffian if and only if it is subcanonical. In the case when $n=4 k$, the last statement is not true; however, there is another structure theorem (see [16]).

The nongeneral type subcanonical threefolds in $\mathbb{P}^{6}$ are either well understood Fano threefolds or threefolds with trivial canonical class. A very natural class of varieties among varieties with trivial canonical class are Calabi-Yau threefolds, i.e., smooth threefolds $X$ with $K_{X}=0$ and $H^{1}\left(X, \mathcal{O}_{X}\right)=0$. In the paper, we shall sometimes also consider singular Calabi-Yau threefolds by which we mean complex projective threefolds with Gorenstein singularities, $\omega_{X}=0$ and with $h^{1}\left(\mathcal{O}_{X}\right)=0$.

For Calabi-Yau threefolds, the theory of Pfaffians is more specific. For instance, Schreyer, following [37], shows that if $X$ is Pfaffian, $h^{i}\left(\mathcal{O}_{X}\right)=0$ for $0<i<\operatorname{dim} X$ and $E$ is any vector bundle such that there exists $\sigma \in H^{0}\left(\bigwedge^{2} E(t)\right)$ with $X=\operatorname{Pf}(\sigma)$ then, keeping the notation above, $E(-s)$ appears as a sum of the sheafified first syzygy module $S y z^{1}(H R(X))$ of the Hartshorne-Rao module $H R(X)=\oplus_{k=1}^{N} H^{1}\left(\mathcal{I}_{X}(k)\right.$ ) (seen as a $\mathfrak{S}=\mathbb{C}\left[x_{0}, \ldots, x_{6}\right]$ module) and a sum of line bundles. If we add the assumption that $X$ has trivial canonical class then, by considering an appropriate twist, we can choose a bundle $E$ such that $t=1$ and $s=3$. More precisely, if $X$ is a Calabi-Yau threefold, then there exists a bundle $E$ of rank $2 u+1$ such that $3=c_{1}(E)+u$ and $X=\operatorname{Pf}(\sigma)$ for some $\sigma \in H^{0}\left(\bigwedge^{2} E(1)\right)$. Moreover, if we denote by $M$ the Hartshorne-Rao module of $X$ with gradation shifted by 3 , then the chosen bundle $E$ is obtained as a sum of $S y z^{1}(M)$ with a sum of line bundles.

Let us point out that all threefolds can be smoothly projected to $\mathbb{P}^{7}$. It is, moreover, known from [4, Remark 11] that Calabi-Yau threefolds embedded in $\mathbb{P}^{5}$ are complete intersections; either of two cubics, or of a quadric and a quartic, or of a quintic and a hyperplane. Having this in mind, we study nondegenerate Calabi-Yau threefolds, i.e., such Calabi-Yau threefolds which are not contained in any hyperplane.

Nondegenerate Calabi-Yau threefolds in $\mathbb{P}^{6}$ were already studied in $[7,8,23,31,34,35]$, and [25], where examples of degree $12 \leq d \leq 17$ were constructed. It is not hard to see 
that the degree of such a threefold is bounded between 11 and 41 (see Corollary 2.2) but we expect a sharper bound (see [24]). Okonek proposed the following problem:

Problem 1.1 Classify the Calabi-Yau threefolds in $\mathbb{P}^{6}$.

The central result of the paper is a full classification of quasi-Buchsbaum Calabi-Yau threefolds in $\mathbb{P}^{6}$, i.e., Calabi-Yau threefolds in $\mathbb{P}^{6}$ such that their higher cohomology modules have trivial structure (see Definition 3.1). The classification is given in Theorem 3.2. The proof that this classification includes all Calabi-Yau threefolds in $\mathbb{P}^{6}$ of degree $d \leq 14$ and a classification of Calabi-Yau threefolds contained in 5-dimensional quadrics is our main result. The classification is given by providing a list of vector bundles $\left\{E_{i}\right\}_{i \in I}$ such that the considered Calabi-Yau threefolds are exactly the smooth threefolds which appear as Pfaffians $\operatorname{Pf}(\sigma)$ for some $\sigma \in H^{0}\left(\bigwedge^{2} E_{i}(1)\right)$ and $i \in I$. Let us point out that our list contains two distinct vector bundles corresponding to degree 14 Calabi-Yau threefolds.

In Sect. 2, we prove basic general results concerning the classification of Calabi-Yau threefolds in $\mathbb{P}^{6}$. In particular, we observe that a Calabi-Yau threefolds in $\mathbb{P}^{6}$ must be linearly normal. We, moreover, prove the finiteness of the classification problem 1.1.

Theorem 3.2 is the main theorem of Sect. 3. It presents the classification of Calabi-Yau threefolds that are quasi-Buchsbaum. As a consequence, we find that the examples that are arithmetically Cohen-Macaulay (see Definition 3.1) are of degrees $12 \leq d \leq 14$.

In Sect. 4, we classify Calabi-Yau threefolds contained in quadrics in terms of degree. More precisely, we prove the following theorem.

Theorem 1.2 If $\left(X, Q_{5}^{r}\right)$ is a pair consisting of a nondegenerate Calabi-Yau threefold $X \subset$ $\mathbb{P}^{6}$ of degree $d_{X}$ and a 5-dimensional quadric $Q_{5}^{r}$ of corank $r$ in $\mathbb{P}^{6}$ such that $X \subset Q_{5}^{r}$, then $r \leq 2$ and $12 \leq d_{X} \leq 14$.

For the proof, we consider case by case the possible coranks of the quadrics containing the Calabi-Yau threefolds. For low corank quadrics (i.e., $r=0,1,2$ ), we consider hyperplane sections of our Calabi-Yau threefold and so work with canonically embedded surfaces of general type; see [10] for more information on such surfaces. For instance, on a smooth quadric in $\mathbb{P}^{5}$ containing a canonically embedded surface of general type $S$, we can apply the double-point formula to get the bound for the degree $d_{S}$ of $S$ to be $12 \leq d_{S} \leq 14$. Inputting an additional assumption on $S$, stating that it is a section of some Calabi-Yau threefold $X$ contained in a smooth quadric in $\mathbb{P}^{6}$, leads to the result $d_{X}=d_{S}=12$ or $d_{X}=d_{S}=14$. For canonically embedded surfaces of general type contained in quadrics of rank 5 in $\mathbb{P}^{5}$, we obtain the same bound $12 \leq d \leq 14$ working on the resolution of this quadric. The latter resolution is the projectivization of a vector bundle of rank 2. The last step is the proof that there are no Calabi-Yau threefolds contained in quadrics of corank $\geq 3$ in $\mathbb{P}^{6}$. It is worth noticing that there is no similar result for canonically embedded surfaces of general type contained in quadrics of corank $\geq 2$ in $\mathbb{P}^{5}$. In particular, we present, in Propositions 4.9, 4.11 examples of nodal Calabi-Yau threefolds contained in quadrics of rank 4 which have degree 11 and 15. Their general hyperplane sections are canonical surfaces of respective degrees 11 and 15 which are contained in 4-dimensional quadrics of rank 4.

The classification of Calabi-Yau threefolds of degree $d \leq 14$ in $\mathbb{P}^{6}$ in terms of vector bundles associated with them by the Pfaffian construction is completed in Sects. 5 and 6 . More precisely, we prove that all Calabi-Yau threefolds of degree at most 14 in $\mathbb{P}^{6}$ are quasi-Buchsbaum and use the classification of the latter threefolds contained in Sect. 3 (see Theorem 3.2).

By Theorem 1.2, the classification of Calabi-Yau threefolds of degree $d \leq 14$ in $\mathbb{P}^{6}$ provides also a classification of all Calabi-Yau threefolds contained in 5-dimensional quadrics. 
We, moreover, observe that there are two types of Calabi-Yau threefolds of degree $d=14$. Calabi-Yau threefolds of the first type are not contained in any quadric whereas Calabi-Yau threefolds of the second type are.

Finally, in Sect. 7, we perform a classification of Calabi-Yau threefolds of degree $d \leq 14$ in $\mathbb{P}^{6}$ up to deformation. Since this type of classification is weaker than the classification in terms of vector bundles and stronger than the classification by degree, the only remaining ingredient is the proof that there is a unique maximal flat family of Calabi-Yau threefolds of degree 14. Throughout the paper, we study three families of Calabi-Yau threefolds of degree 14. The first is the family $\mathfrak{C}_{14}$ of degree 14 Calabi-Yau threefolds contained in a smooth quadric $Q_{5}^{0}$. To define it, we think of the smooth 5-dimensional quadric $Q_{5}^{0}$ as a homogenous variety with respect to the standard action of the simple Lie group $\mathbb{G}_{2}$. Then $Q_{5}^{0}$ admits a natural bundle $\mathcal{C}$ called a Cayley bundle which is homogeneous with respect to this action. The family $\mathfrak{C}_{14}$ is the family of all smooth threefolds appearing as zero loci of sections of a twist $\mathcal{C}(3)$. To confirm that the family is not complete, we compute that these threefolds have more deformations then obtained by varying the section of $\mathcal{C}(3)$. In fact, by Corollary 5.4, we deduce that $\mathfrak{C}_{14}$ is part of a larger family $\mathfrak{B}_{14}$ of threefolds given as Pfaffian varieties associated with the bundle $E=\Omega_{\mathbb{P} 6}^{1}(1) \oplus \mathcal{O}_{\mathbb{P} 6}(1)$. Then we prove a technical result (Proposition 7.2) on deformation of Pfaffian varieties implying that any threefold $B_{14} \in \mathfrak{B}_{14}$ appears as a smooth degeneration of the family $\mathfrak{T}_{14}$ of Calabi-Yau threefolds defined by $6 \times 6$ Pfaffians of alternating $7 \times 7$ matrices of linear forms. This proves that all families of Calabi-Yau threefolds of degree 14 which appear in the classification of Sect. 6 are in the same component of the Hilbert scheme.

Corollary 1.3 There is one family of Calabi-Yau threefolds in $\mathbb{P}^{6}$ in each degree $d \leq 14$.

Families of threefolds $\mathfrak{B}_{14}$ and $\mathfrak{B}_{15}$ of degrees 14 and 15 , respectively, that we consider in this paper were already constructed in [7]; however, our results stay in contradiction with [7, Prop 4.3.] and the results in subsections 4.2.2 and 4.2.3 therein. In particular, we prove that both the examples of degree 14 and 15 are flat deformations of families of Calabi-Yau threefolds constructed in [35]. This means that if to each member of a family of deformations of Calabi-Yau threefolds in $\mathbb{P}^{6}$ we associate the minimal degree of hypersurfaces containing it then, unlike in the case of complete intersections, this number can drop for special members. We, moreover, prove that the examples of degree 15 constructed in [7] are not smooth but admits three ordinary double points.

\section{Preliminaries}

Let us first discuss some general properties of Calabi-Yau threefolds embedded in $\mathbb{P}^{6}$. We call such a threefold nondegenerate if it is not contained in any hyperplane. The degenerate Calabi-Yau threefolds (those which are not nondegenerate) in $\mathbb{P}^{6}$ are known to be complete intersections either $X_{3,3} \subset \mathbb{P}^{5} \subset \mathbb{P}^{6}$ or $X_{2,4} \subset \mathbb{P}^{5} \subset \mathbb{P}^{6}$ or $X_{5} \subset \mathbb{P}^{4} \subset \mathbb{P}^{6}$ (see [4, Remark 11]).

Proposition 2.1 Let $X \subset \mathbb{P}^{6}$ be a Calabi-Yau threefold; then $X$ is linearly normal, i.e., the natural restriction map $H^{0}\left(\mathcal{O}_{\mathbb{P}^{6}}(1)\right) \rightarrow H^{0}\left(\mathcal{O}_{X}(1)\right)$ is surjective.

Proof It follows from [18, Theorem 2.1] that there are only three families of nonlinearly normal threefolds in $\mathbb{P}^{6}$. These families have degrees 6,7 and 8 , respectively, and cannot be Calabi-Yau threefolds. 
Next, we show an a priori bound on the degree of the Calabi-Yau threefolds contained in $\mathbb{P}^{6}$.

Corollary 2.2 The degree d of a nondegenerate Calabi-Yau threefold $X \subset \mathbb{P}^{6}$ is bounded between $11 \leq d \leq 41$

Proof Observe that a generic hyperplane section $S \subset X$ is a canonically embedded surface of general type. It was already remarked in [35] that using the Castelnuovo inequality for surfaces of general type, we can deduce that $d \geq 11$. Next, from the Riemann-Roch theorem for line bundles on $X$ and Proposition 2.1, we deduce that $\frac{1}{12} S . c_{2}(X)+\frac{1}{6}==h^{0}\left(\mathcal{O}_{X}(1)\right)=$ $\chi\left(\mathcal{O}_{X}(1)\right)=7 d$. It is a classical result on Calabi-Yau threefolds, contained in [27], that $H . c_{2}(X) \geq 0$ for every ample divisor $H$ on $X$. Thus, we infer $7 \leq \frac{1}{6} d$.

Moreover, from [27], we also know that, for any ample divisor $H$, we have $H . c_{2}(X)=0$ if and only if $X$ is a finite étale quotient of an abelian threefold (this implies in particular $c_{2}(X)=0$ ). Let us now show that $d=42$ is impossible. By the above, in this case, it is enough to consider Calabi-Yau threefolds with trivial $c_{2}(X)$. Those were classified in [28, Theorem 0.1]. There are two possibilities, and in each of them, we have $\chi_{\text {top }}(X)=0$. On the other hand, from the double-point formula (cf. [35]) we get

$$
\chi_{\text {top }}(X)=-d^{2}+49 d-588=-(42)^{2}+49 \cdot 42-588 \neq 0 .
$$

We thus obtain a contradiction proving that $d \neq 42$ and in consequence $d \leq 41$.

Remark 2.3 It is a natural problem to ask whether other smooth threefolds with $K_{X}=0$ (i.e., without assuming that $h^{1}\left(\mathcal{O}_{X}\right)=0$ ) can be embedded in $\mathbb{P}^{6}$. Note that, in [36], it is proven that there are no Abelian threefolds in $\mathbb{P}^{6}$.

Corollary 2.2 implies, in particular, that there is a finite number of families of Calabi-Yau threefolds in $\mathbb{P}^{6}$; three families of degenerate examples and a finite number of nondegenerate ones. Indeed, by the Riemann-Roch theorem, $H . c_{2}(X)$ and the degree $H^{3}$ determine the Hilbert polynomial of a polarized Calabi-Yau threefold $(X, H)$. Moreover, if $(X, H)$ is a Calabi-Yau threefold in $\mathbb{P}^{6}$ polarized by its hyperplane section then, again by the RiemannRoch theorem, $H . c_{2}(X)$ is determined by $H^{3}$ and $h^{0}\left(\mathcal{O}_{X}(H)\right)=7$. It follows that the Hilbert polynomial of $X$ is determined by the degree of $X$. Hence, in each degree, there is a finite number of families. This means that having a bound on the degree implies finiteness.

A slightly sharper bound on the degree could be obtained for Calabi-Yau threefolds with $\operatorname{rk} \operatorname{Pic}(X)=h^{1,1}(X)=1$. In this case, using the double-point formula 2.1, we obtain

$$
2 \geq 2\left(h^{1,1}(X)-h^{1,2}(X)\right)=\chi_{t o p}(X)=-d^{2}+49 d-588 .
$$

We then infer that either $d \leq 21$ or $d \geq 28$. However, let us point out that there exist examples of Calabi-Yau threefolds $X \subset \mathbb{P}^{6}$ with $h^{1,1}(X)>1$ (see [24, Corollary 5.9.]).

\section{Quasi-Buchsbaum Calabi-Yau threefolds}

Recall the following definitions.

Definition 3.1 Let $X \subset \mathbb{P}^{n}$ be a subvariety of positive dimension in a projective space. Let us, for each $i \in \mathbb{N}_{\geq 0}$, denote by $H_{*}^{i}\left(\mathcal{I}_{X}\right)$ the $i$-th cohomology module $\bigoplus_{j \in \mathbb{Z}} H^{i}\left(\mathbb{P}^{n}, \mathcal{I}_{X}(j)\right)$. We say that $X$ is arithmetically Cohen-Macaulay (aCM for short) if and only if $H_{*}^{i}\left(\mathcal{I}_{X}\right)=0$ for $1 \leq i \leq \operatorname{dim}(X)-1$. Moreover, $X$ is called quasi-Buchsbaum if and only if, for 
$1 \leq i \leq \operatorname{dim}(X)-1$, we have: $H_{*}^{i}\left(\mathcal{I}_{X}\right)$ is annihilated by the maximal ideal of the structure ring of $\mathbb{P}^{n}$. Finally, $X$ is arithmetically Buchsbaum if each of its linear sections is quasiBuchsbaum.

It is part of the mathematical folklore that the aCM Calabi-Yau threefolds in $\mathbb{P}^{6}$ are only the ones listed in [35] up to degree 14. However, since we have not found a proper proof of this fact in the literature, we provide it below as a consequence of a more general result which will be important for the rest of the paper. More precisely, we provide a classification of all quasi-Buchsbaum Calabi-Yau threefolds in $\mathbb{P}^{6}$. In particular, this also gives a classification of all arithmetically Buchsbaum Calabi-Yau threefolds in $\mathbb{P}^{6}$.

Theorem 3.2 Let $X$ be a quasi-Buchsbaum Calabi-Yau threefold in $\mathbb{P}^{6}$. Then $X=P f(\varphi)$ for some $\varphi \in H^{0}\left(\mathbb{P}^{6}, \bigwedge^{2} E(1)\right)$ where $E$ is a vector bundle such that one of the followings holds:

(1) $E=\bigoplus_{i=1}^{2 u+1} \mathcal{O}_{\mathbb{P}^{6}}\left(a_{i}\right)$ with:

(a) $u=1, a_{1}=-2, a_{2}=2, a_{3}=2$, and $X$ is a complete intersection of type 1,1,5;

(b) $u=1, a_{1}=-1, a_{2}=1, a_{3}=2$, and $X$ is a complete intersection of type 1,2,4;

(c) $u=1, a_{1}=0, a_{2}=0, a_{3}=2$, and $X$ is a complete intersection of type 1,3,3;

(d) $u=1, a_{1}=0, a_{2}=1, a_{3}=1$, and $X$ is a complete intersection of type 2,2,3;

(e) $u=2, a_{i}=0$, for $i \in\{1 \ldots 4\}, a_{5}=1$, and $X$ is a degree 13 Calabi-Yau threefold described in [35];

(f) $u=3, a_{i}=0$, for $i \in\{1 \ldots 7\}$, and $X$ is a degree 14 Calabi-Yau threefold described in $[31,35]$;

(2) $E=\Omega_{\mathbb{P} 6}^{1}(1) \oplus \bigoplus_{i=1}^{2 v+1} \mathcal{O}_{\mathbb{P}^{6}}\left(a_{i}\right)$ with:

(a) $v=0, a_{1}=1$, and $X$ is a degree 14 Calabi-Yau threefold from the family $\mathfrak{B}_{14}$ described in [7] (see also Example 5.3);

(b) $v=1, a_{1}=0, a_{2}=0, a_{3}=0$, and $X$ is a degree 15 Calabi-Yau threefold described in [35].

Proof Take $X$ a quasi-Buchsbaum Calabi-Yau threefold in $\mathbb{P}^{6}$. Then, by definition, the Hartshorne-Rao module $H R(X)=H_{*}^{1}\left(\mathcal{I}_{X}\right)$ is annihilated by the maximal ideal of the structure ring of $\mathbb{P}^{6}$. It follows that the resolution of $H R(X)$ is given by a direct sum of twisted Koszul complexes. Thus, the sheafification $S y z^{1}(H R(X))$ of the first syzygy module of $H R(X)$ is $\bigoplus_{i=1}^{n_{b}} \Omega^{1}\left(b_{i}-2\right)$ for some $b_{1} \ldots b_{n_{b}} \in \mathbb{Z}$. We, moreover, claim that $b_{i} \leq 0$ for $i=1 \ldots n_{b}$. Indeed, from Proposition 2.1 and the exact sequence:

$$
0 \rightarrow \mathcal{I}_{X}(q) \rightarrow \mathcal{O}_{\mathbb{P} 6}(q) \rightarrow \mathcal{O}_{X}(q) \rightarrow 0 .
$$

we have $H^{1}\left(\mathcal{I}_{X}(q)\right)=0$ for $q \leq 1$. To prove the claim, it is now enough to observe that in the above notation, we have $H^{1}\left(\mathcal{I}_{X}\left(2-b_{i}\right)\right)>0$ for $i=1 \ldots n_{b}$.

Let, now, $M$ be the module obtained by shifting the gradation in the Hartshorne-Rao module $H R(X)$ by 3. As observed in the introduction, there exists a vector bundle $E=$ $\operatorname{Syz}^{1}(M) \oplus \bigoplus_{j=1}^{n_{a}} \mathcal{O}_{\mathbb{P} 6}\left(a_{i}\right)$ with $a_{1} \ldots a_{n_{a}} \in \mathbb{Z}$ and a section $\varphi \in H^{0}\left(\mathbb{P}^{6}, \bigwedge^{2} E(1)\right)$ such that $X=P f(\varphi)$. Without loss of generality, we assume that $E$ is a bundle that has minimal rank among bundles for which there exists such a $\varphi \in H^{0}\left(\mathbb{P}^{6}, \bigwedge^{2} E(1)\right)$ that $X=P f(\varphi)$. The rank of the bundle $E$ is $2 u+1$ for some $u \in \mathbb{N}$. Observe that there is a decomposition

$$
E=\bigoplus_{i=1}^{n_{b}} \Omega^{1}\left(b_{i}+1\right) \oplus \bigoplus_{j=1}^{n_{a}} \mathcal{O}_{\mathbb{P}^{6}}\left(a_{i}\right) \text {. }
$$


We fix such a decomposition for the rest of the proof with $a_{i}$ and $b_{i}$ arranged in an increasing order. The bundles appearing in this decomposition, treated both as subbundles and as quotient bundles, will be called components of $E$. We also have an induced decomposition

$$
\begin{aligned}
E^{*}(-1)= & \bigoplus_{i=1}^{n_{b}}\left(\Omega^{1}\left(b_{i}+1\right)\right)^{*}(-1) \oplus \bigoplus_{j=1}^{n_{a}} \mathcal{O}_{\mathbb{P}^{6}}\left(-a_{i}-1\right) \\
& =\bigoplus_{i=1}^{n_{b}}\left(\Omega^{5}\left(5-b_{i}\right)\right) \oplus \bigoplus_{j=1}^{n_{a}} \mathcal{O}_{\mathbb{P}^{6}}\left(-a_{i}-1\right) .
\end{aligned}
$$

One can, now, think of $\varphi$ as of a matrix consisting of blocks of the following types

- $\varphi_{i, j}^{a}=\left.\pi_{j}^{a} \circ \varphi\right|_{\mathcal{O}_{\mathbb{P} 6}\left(-a_{i}-1\right)}: \mathcal{O}_{\mathbb{P}^{6}}\left(-a_{i}-1\right) \rightarrow \mathcal{O}_{\mathbb{P}^{6}}\left(a_{j}\right)$, for $i, j \in\left\{1, \ldots, n_{a}\right\}$;

- $\varphi_{i, j}^{a, b}=\left.\pi_{j}^{b} \circ \varphi\right|_{\mathcal{O}_{\mathbb{P} 6}\left(-a_{i}-1\right)}: \mathcal{O}_{\mathbb{P}^{6}}\left(-a_{i}-1\right) \rightarrow \Omega_{\mathbb{P}^{6}}^{1}\left(b_{j}+1\right)$, for $i \in\left\{1, \ldots, n_{a}\right\}, j \in$ $\left\{1, \ldots, n_{b}\right\}$

- $\varphi_{i, j}^{a, b}=\left.\pi_{j}^{a} \circ \varphi\right|_{\Omega_{\mathbb{P} 6}^{5}\left(6-b_{i}\right)}: \Omega_{\mathbb{P}^{6}}^{5}\left(5-b_{i}\right) \rightarrow \mathcal{O}_{\mathbb{P}^{6}}\left(a_{j}\right)$ for $i \in\left\{1, \ldots, n_{b}\right\}, j \in\left\{1, \ldots, n_{a}\right\}$;

- $\varphi_{i, j}^{b}=\left.\pi_{j}^{b} \circ \varphi\right|_{\Omega_{\mathbb{P}^{6}}^{5}\left(6-b_{i}\right)}: \Omega_{\mathbb{P}^{6}}^{5}\left(5-b_{i}\right) \rightarrow \Omega_{\mathbb{P}^{6}}^{1}\left(b_{j}+1\right)$ for $i, j \in\left\{1, \ldots, n_{b}\right\}$;

where $\pi_{j}^{a}$ and $\pi_{j}^{b}$ are the projections onto the components of $E$, i.e., $\mathcal{O}_{\mathbb{P}^{6}}\left(a_{j}\right)$ and $\Omega_{\mathbb{P}^{6}}^{1}\left(b_{j}+1\right)$, respectively, for each $j$. We then have $\varphi_{i, j}^{a}=-\left(\varphi_{j, i}^{a}\right)^{*}, \varphi_{i, j}^{a, b}=-\left(\varphi_{j, i}^{b, a}\right)^{*}$ and $\varphi_{i, j}^{b}=-\left(\varphi_{j, i}^{b}\right)^{*}$. Equivalently, we have a decomposition:

$$
\begin{aligned}
H^{0}\left(\bigwedge^{2} E(1)\right)= & \bigoplus_{1 \leq i<j \leq n_{a}} H^{0}\left(\mathcal{O}_{\mathbb{P}^{6}}\left(a_{i}+a_{j}+1\right)\right) \oplus \underset{\substack{i \in\left\{1 \ldots n_{a}\right\} \\
j \in\left\{1 \ldots n_{b}\right\}}}{\bigoplus} H^{0}\left(\Omega_{\mathbb{P}^{6}}^{1}\left(a_{i}+b_{j}+2\right)\right) \\
& \oplus \bigoplus_{1 \leq i<j \leq n_{b}} H^{0}\left(\Omega_{\mathbb{P}^{6}}^{1} \otimes \Omega_{\mathbb{P}^{6}}^{1}\left(b_{i}+b_{j}+3\right)\right) \oplus \bigoplus_{i=1}^{n_{b}} H^{0}\left(\Omega_{\mathbb{P}^{6}}^{2}\left(2 b_{i}+3\right)\right)
\end{aligned}
$$

and the maps $\varphi_{i, j}^{a}, \varphi_{i, j}^{a, b}, \varphi_{i, j}^{b}, \varphi_{i, i}^{b}$ are identified with the respective components of $\varphi$ in the above decomposition. By abuse of notation, we shall use this identification without further commentary throughout the proof. We shall, moreover, use the notation $\varphi_{E_{1}, E_{2}}$ for the projection of $\varphi$ onto $\left(E_{1} \otimes E_{2}\right)(1)$ for any two subbundles $E_{1}, E_{2} \subset E$ appearing as sums of components of $E$ and for the corresponding map $E_{1}^{*}(-1) \rightarrow E_{2}$.

Our aim is to deduce as many as possible restrictions on the coefficients $a_{i}$ and $b_{i}$ following from the fact that $\varphi$ defines a codimension 3 variety which has trivial canonical class. The first such condition is given by the formula 1.2 for the canonical class of a Pfaffian variety given in the introduction. We have

$$
\omega_{X}=\mathcal{O}_{X}\left(2 u+2 c_{1}(E)-6\right)
$$

which means that $c_{1}(E)+u=3$. Since $c_{1}(E)=\sum_{i=1}^{n_{b}}\left(-1+6 b_{i}\right)+\sum_{j=1}^{n_{a}} a_{j}$, we have the equation

$$
u+\sum_{i=1}^{n_{b}}\left(-1+6 b_{i}\right)+\sum_{j=1}^{n_{a}} a_{j}=3 .
$$

Since, in the above, both negative and positive $a_{j}$ 's may occur, we, so far, still have infinitely many possibilities for the bundle $E$. To reduce this number of possibilities to a finite number, we introduce the invariant $w(F)=c_{1}(F)+\frac{1}{2} \mathrm{rk} F \in \frac{1}{2} \mathbb{Z}$ for each subbundle $F$ of $E$. It is 
clear that $w$ is additive when taking direct sums of bundles. Moreover, in terms of $w$, formula 3.2 takes the form

$$
w(E)=3+\frac{1}{2}
$$

Our aim is to find a natural decomposition of $E$ into a direct sum of as many as possible bundles with positive $w$. We proceed as follows. Let $n_{\text {neg }}^{a}$ be the number of negative $a_{i}$ 's $n_{\text {neg }}^{b}$ the number of negative $b_{i}$ 's and $n_{\text {pos }}$ the number of positive $a_{i}$ 's, finally we set $n_{\text {neg }}:=$ $n_{\text {neg }}^{a}+6 n_{\text {neg. }}^{b}$. Define the sequence $\left(l_{i}^{\prime}\right)_{i \in\left\{1 \ldots n_{\text {neg }}\right\}}$ by

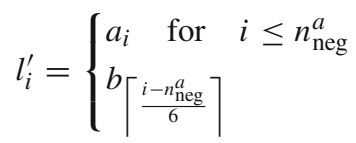

In this way, we get a sequence that contains all negative $a_{i}$ 's and moreover all negative $b_{i}$ 's each occurring 6 times. The reason why we want the $b_{i}$ 's to occur 6 times in our sequence is that in the latter, we shall deal with ranks of suitable components of the decomposition of $E$ and the $b_{i}$ 's represent components of rank 6 . We then rearrange the sequence $\left(l_{i}^{\prime}\right)_{i \in\left\{1 \ldots n_{\text {neg }}\right\}}$ to a weakly increasing sequence and obtain in this way a sequence $\left(l_{i}\right)_{i \in\left\{1 \ldots n_{\text {neg }}\right\}}$. Let us moreover define $\left(k_{i}\right)_{i \in\left\{1 \ldots n_{\text {pos }}\right\}}$ to be the weakly decreasing sequence consisting of positive $a_{i}$ 's.

The key to the proof of Theorem 3.2 is the following lemma.

Lemma 3.3 Under the above notation, either $n_{\text {neg }}=n_{\text {pos }}=0$, or both the following hold:

- $n_{\text {neg }}<n_{\text {pos }}$,

- $l_{i}+k_{i+1} \geq 0$ for each $i \in 1 \ldots n_{\text {neg }}$.

Before we pass to the proof of Lemma 3.3, let us finish the proof of Theorem 3.2 assuming the lemma.

Keeping our notation, each component $F$ of $E$ such that $w(F)<0$ is either a line bundle $\mathcal{O}_{\mathbb{P}^{6}}\left(l_{i(F)}\right)$ for some $i(F) \in\left\{1 \ldots n_{\text {neg }}\right\}$ or a bundle of twisted first differentials $\Omega_{\mathbb{P}^{6}}^{1}\left(l_{i(F)}+1\right)$ with $l_{i(F)}=\cdots=l_{i(F)+5}$ for some $i(F) \in\left\{1 \ldots n_{\text {neg }}\right\}$. Now, to each component $F$ of $E$ with $w(F)<0$, one associates a vector subbundle $A(F)$ of $E$ in the following way.

$$
A(F):= \begin{cases}\mathcal{O}_{\mathbb{P} 6}\left(k_{i(F)+1}\right) & \text { for } F=\mathcal{O}_{\mathbb{P} 6}\left(l_{i(F)}\right) \\ \bigoplus_{j=1}^{6} \mathcal{O}_{\mathbb{P}^{6}}\left(k_{i(F)+j}\right) & \text { for } F=\Omega_{\mathbb{P}^{6}}^{1}\left(l_{i(F)}+1\right)\end{cases}
$$

By Lemma 3.3, the bundle $A(F)$ is well defined for every component $F$ of $E$ with $w(F)<$ 0 and the following holds:

- $A(F)$ is a sum of components of positive degree not involving the component $\mathcal{O}_{\mathbb{P} 6}\left(k_{1}\right)$;

- if $F_{1} \cap F_{2}=0$ then $A\left(F_{1}\right) \cap A\left(F_{2}\right)=0$;

- $\operatorname{rk} A(F)=\operatorname{rk} F$;

- $w(F)+w(A(F))>0$.

Indeed, the only that needs to be checked is the last inequality. If $F=\mathcal{O}_{\mathbb{P}^{6}}\left(l_{i(F)}\right)$, then $w(F)=$ $l_{i(F)}+\frac{1}{2}$ and $w(A(F))=k_{i(F)+1}+\frac{1}{2}$ thus from Lemma 3.3 we get $w(F)+w(A(F)) \geq$ $1=\operatorname{rk} F$. If $F=\Omega_{\mathbb{P}^{6}}^{1}\left(l_{i(F)}+1\right)$ then $w(F)=-1+6 l_{i(F)}+3=\left(\sum_{j=0}^{5} l_{i(F)+j}\right)+2$ and $w(A(F))=\left(\sum_{j=0}^{5} k_{i(F)+j+1}\right)+3$ we conclude again by Lemma 3.3. From the above, we get a decomposition of $E$ :

$$
E=\bigoplus_{\substack{F \text { component of } E \\ w(F)<0}}(F \oplus A(F)) \oplus \bigoplus_{\substack{F \text { component of } E \\ w(F)>0 \\ F \cap \bigoplus_{w(G)<0} A(G)=0}} F
$$


into bundles with positive $w$ (observe that $w(F) \neq 0$ when $F$ is a component of $E$ ) including the line bundle of maximal degree $\mathcal{O}_{\mathbb{P} 6}\left(k_{1}\right)$. Recall moreover that in order to obtain a Calabi-Yau threefold these positive values of $w$ in this decomposition must add up to $3+\frac{1}{2}$. We then easily list all possibilities. Indeed, we have:

(1) $b_{i}=0$ for $i \in\left\{1 \ldots n_{b}\right\}$, because for any $c \leq 0$, by the above computation involving Lemma 3.3 we have $w\left(\Omega_{\mathbb{P} 6}^{1}(c) \oplus A\left(\Omega_{\mathbb{P} 6}^{1}(c)\right)\right) \geq 5>3+\frac{1}{2}$;

(2) $n_{b} \leq 1$, because since $b_{i} \leq 0$ in the opposite case in the decomposition we would have two components of the form $\Omega_{\mathbb{P} 6}^{1}(1)$ but $2 w\left(\Omega_{\mathbb{P} 6}^{1}(1)\right)=4>3+\frac{1}{2}$;

(3) if $n_{b}=1$ then there is no negative $a_{i}$, because otherwise $w\left(\Omega_{\mathbb{P} 6}^{1}(1) \oplus \mathcal{O}_{\mathbb{P} 6}\left(l_{1}\right) \oplus\right.$ $\left.\mathcal{O}_{\mathbb{P} 6}\left(k_{1}\right) \oplus \mathcal{O}_{\mathbb{P} 6}\left(k_{2}\right)\right) \geq 4+\frac{1}{2}$

(4) if $n_{b}=0$ then $l_{1} \geq-2$, because when $l_{1} \leq-3$, we have $w\left(\mathcal{O}_{\mathbb{P}^{6}}\left(l_{1}\right) \oplus \mathcal{O}_{\mathbb{P}^{6}}\left(k_{1}\right) \oplus\right.$ $\left.\mathcal{O}_{\mathbb{P}^{6}}\left(k_{2}\right)\right) \geq 4+\frac{1}{2}$

(5) if $l_{1}=-2$ then $E=\mathcal{O}_{\mathbb{P}^{6}}(-2) \oplus \mathcal{O}_{\mathbb{P}^{6}}(2)$, because $w\left(\mathcal{O}_{\mathbb{P}^{6}}\left(l_{1}\right) \oplus \mathcal{O}_{\mathbb{P}^{6}}\left(k_{1}\right) \oplus \mathcal{O}_{\mathbb{P}^{6}}\left(k_{2}\right)\right) \geq$ $3+\frac{1}{2}$ and equality holds only if $k_{1}=k_{2}=2$;

(6) if $l_{1}=l_{2}=-1$ then $E=3 \mathcal{O}_{\mathbb{P} 6}(1) \oplus 2 \mathcal{O}_{\mathbb{P} 6}(-1)$, because $w\left(\mathcal{O}_{\mathbb{P} 6}\left(l_{1}\right) \oplus \mathcal{O}_{\mathbb{P} 6}\left(l_{2}\right) \oplus\right.$ $\left.\mathcal{O}_{\mathbb{P} 6}\left(k_{1}\right) \oplus \mathcal{O}_{\mathbb{P} 6}\left(k_{2}\right) \oplus \mathcal{O}_{\mathbb{P}^{6}}\left(k_{3}\right)\right) \geq 3+\frac{1}{2}$ and equality holds only if $k_{1}=k_{2}=k_{3}=1$;

(7) if $l_{1}=-1$ and all remaining $a_{i}$ are nonnegative then we have two possibilities:

(a) $E=\mathcal{O}_{\mathbb{P}^{6}}(-1) \oplus \mathcal{O}_{\mathbb{P}^{6}}(1) \oplus \mathcal{O}_{\mathbb{P}^{6}}(2)$

(b) $E=\mathcal{O}_{\mathbb{P}^{6}}(-1) \oplus 2 \mathcal{O}_{\mathbb{P}^{6}}(1) \oplus 2 \mathcal{O}_{\mathbb{P}^{6}}$ and the constant terms in $\varphi$ are 0

(8) if $n_{b}=0$ and all $a_{i}$ are nonnegative we have 4 possibilities for $E$ as in the assertion.

To conclude, we need to exclude two cases which do not appear in the assertion:

- $E=3 \mathcal{O}_{\mathbb{P}^{6}}(1) \oplus 2 \mathcal{O}_{\mathbb{P}^{6}}(-1)$; we shall see in Proposition 4.9 that Pfaffian varieties associated with this bundle are always singular.

- $E=\mathcal{O}_{\mathbb{P}^{6}}(-1) \oplus 2 \mathcal{O}_{\mathbb{P} 6}(1) \oplus 2 \mathcal{O}_{\mathbb{P} 6}$ and the constant terms in $\varphi \in \bigwedge^{2} E(1)$ are 0 ; we easily see that $\operatorname{Pf}(\varphi)$ either does not exist (i.e., the degeneracy locus is of codimension $\leq 2$ ) or must be contained in a hyperplane.

To complete the proof of Theorem 3.2, we, hence, need only to prove Lemma 3.3 and Proposition 4.9.

Proof of Lemma 3.3 If $n_{n e g}=0$, then the assertion is trivial. Assume, hence, by contradiction that $n_{\text {neg }}>0$ and that there exists $i \in\left\{1 \ldots n_{\text {neg }}\right\}$ such that $i+1>n_{\text {pos }}$ or that $k_{i+1}+l_{i}<0$. Let

$$
E_{1}=\bigoplus_{\left\{j \mid a_{j} \leq l_{i}\right\}} \mathcal{O}_{\mathbb{P}^{6}}\left(a_{j}\right) \oplus \bigoplus_{\left\{j \mid b_{j} \leq l_{i}\right\}} \Omega_{\mathbb{P}^{6}}^{1}\left(1+b_{j}\right)
$$

considered as a subbundle of $E$. Observe that, by definition of the sequence $\left(l_{i}\right)_{i \in\left\{1 \ldots n_{\text {neg }}\right\}}$, we have rk $E_{1} \geq i$. Let, moreover,

$$
E_{2}= \begin{cases}\bigoplus_{\left\{j \mid a_{j} \leq k_{i+1}\right\}} \mathcal{O}_{\mathbb{P}^{6}}\left(a_{j}\right) \oplus \bigoplus_{\left\{j \mid b_{j} \leq k_{i+1}\right\}} \Omega_{\mathbb{P}^{6}}^{1}\left(1+b_{j}\right) & \text { when } i+1 \leq n_{\text {pos }} \\ \bigoplus_{\left\{j \mid a_{j} \leq 0\right\}} \mathcal{O}_{\mathbb{P}^{6}}\left(a_{j}\right) \oplus \bigoplus_{\left\{j \mid b_{j} \leq 0\right\}} \Omega_{\mathbb{P}^{6}}^{1}\left(1+b_{j}\right) & \text { when } i+1>n_{\text {pos }}\end{cases}
$$

also considered as a subbundle of $E$. Similarly as for $E_{1}$ we have rk $E_{2} \geq 2 u+1-n_{\text {pos }}$ when $i+1>n_{\text {pos }}$ and rk $E_{2} \geq 2 u+1-i$. In any case:

$$
\text { rk } E_{1}+\mathrm{rk} E_{2} \geq \mathrm{rk} E=2 u+1 .
$$

We also clearly have $E_{1} \subset E_{2}$. Moreover, the following lemma proves that $\varphi_{E_{1}, E_{2}}=0$. 
Lemma 3.4 In the notation of the proof of Theorem 3.2, we have:

- $\varphi_{i, j}^{a}=0$ when $a_{i}+a_{j} \leq-1$

- $\varphi_{i, j}^{a, b}=0$ and $\varphi_{j, i}^{b, a}=0$ when $a_{i}+b_{j} \leq-1$

- $\varphi_{i, j}^{b}=0$ when $b_{i}+b_{j} \leq 0$

Proof Using the following vanishing

- $\operatorname{Hom}\left(\mathcal{O}_{\mathbb{P} 6}(-a-1), \mathcal{O}_{\mathbb{P}^{6}}(b)\right)=H^{0}\left(\mathcal{O}_{\mathbb{P} 6}(b+a+1)\right)=0$ when $a+b \leq-2$

- $\operatorname{Hom}\left(\mathcal{O}_{\mathbb{P} 6}(-a-1), \Omega_{\mathbb{P} 6}^{1}(b+1)\right)=H^{0}\left(\Omega_{\mathbb{P 6}}^{1}(a+b+2)\right)=0$ when $a+b \leq-1$

- $\operatorname{Hom}\left(\left(\Omega_{\mathbb{P}^{6}}^{1}\right)^{*}(-a-2), \Omega_{\mathbb{P}^{6}}^{1}(b+1)\right)=H^{0}\left(\Omega_{\mathbb{P}^{6}}^{2}(a+b+3)\right)=0$ when $a+b \leq-1$

we get

- $\varphi_{i, j}^{a}=0$ when $a_{i}+a_{j} \leq-2$

- $\varphi_{i, j}^{a, b}=0$ and $\varphi_{j, i}^{b, a}=0$ when $a_{i}+b_{j} \leq-1$

- $\varphi_{i, j}^{b}=0$ when $b_{i}+b_{j} \leq 0$

It remains to prove that $\varphi_{i, j}^{a}=0$ when $a_{i}+a_{j}=-1$. Assume the contrary, then $\varphi_{i, j}^{a}$, for some $i, j \in\{1 \ldots l\}$, is a nonzero section of $\mathcal{O}_{\mathbb{P}^{6}}$ thus a nonzero constant which we assume to be 1 by rescaling. Denote by $E^{\prime}$ the subbundle of $E$ such that $E=E^{\prime} \oplus \mathcal{O}_{\mathbb{P}^{6}}\left(a_{i}\right) \oplus \mathcal{O}_{\mathbb{P}^{6}}\left(a_{j}\right)$. The section $\varphi$ is then decomposed as a sum

$$
\begin{aligned}
& \varphi=\left(1, \varphi_{\mathcal{O}_{\mathbb{P} 6}\left(a_{i}\right), E^{\prime}}, \varphi_{\mathcal{O}_{\mathbb{P} 6}\left(a_{j}\right), E^{\prime}}, \varphi_{E^{\prime}, E^{\prime}}\right) \\
& \in H^{0}\left(\mathcal{O}_{\mathbb{P}^{6}} \oplus E^{\prime}\left(a_{i}+1\right) \oplus E^{\prime}\left(a_{j}+1\right) \oplus \bigwedge^{2} E^{\prime}(1)\right)
\end{aligned}
$$

Consider

$$
\varphi^{\prime}=\left(1,0,0, \varphi_{E^{\prime}, E^{\prime}}+\left(\varphi_{\mathcal{O}_{\mathbb{P} 6}\left(a_{i}\right), E^{\prime}} \wedge \varphi_{\mathcal{O}_{\mathbb{P} 6}\left(a_{j}\right), E^{\prime}}\right)\right) .
$$

We claim that $\operatorname{Pf}(\varphi)=\operatorname{Pf}\left(\varphi^{\prime}\right)=\operatorname{Pf}(\psi)$, where $\psi=\varphi_{E^{\prime}, E^{\prime}}+\left(\varphi_{\mathcal{O}_{\mathbb{P} 6}\left(a_{i}\right), E^{\prime}} \wedge \varphi_{\mathcal{O}_{\mathbb{P} 6}\left(a_{j}\right), E^{\prime}}\right) \in$ $H^{0}\left(\bigwedge^{2} E^{\prime}(1)\right)$. Indeed, $\operatorname{Pf}(\varphi)=\operatorname{Pf}\left(\varphi^{\prime}\right)$ follows from the fact that locally under a trivialization of $E$ respecting the decomposition $E=E^{\prime} \oplus \mathcal{O}_{\mathbb{P}^{6}}\left(a_{i}\right) \oplus \mathcal{O}_{\mathbb{P}^{6}}\left(a_{j}\right)$ we have $\varphi^{\prime}$ is obtained by row and column operations from $\varphi$. On the other hand, the equality $\operatorname{Pf}\left(\varphi^{\prime}\right)=\operatorname{Pf}(\psi)$ is clear. The claim being proven, we get a contradiction with the minimality of $E$. This shows that $\varphi_{i, j}^{a}=0$ when $a_{i}+a_{j}=-1$ and completes the proof.

We conclude the proof of Lemma 3.3 by obtaining a contradiction of the above with the following lemma.

Lemma 3.5 Let $E_{1} \subset E_{2} \subset E$ be subbundles of $E$ given by some sums of its components. Consider $\varphi_{E_{1}, E_{2}}: E_{1}^{*}(-1) \rightarrow E_{2}$ as defined above. If $\varphi_{E_{1}, E_{2}}=0$ then $\operatorname{rk}\left(E_{1}\right)+\operatorname{rk}\left(E_{2}\right)<$ $2 u+1$.

Proof Consider the map $\varphi_{E_{1}, E_{2}^{c}}$, where $E_{2}^{c}$ is the subbundle of $E$ being the sum of those components of $E$ which are not contained in $E_{2}$. Under our assumptions, we clearly have $D_{2 u-1}(\varphi) \supset D_{\left(\mathrm{rk}\left(E_{1}\right)-2\right)}\left(\varphi_{\left.E_{1}, E_{2}^{c}\right)}\right.$. Now, since $D_{2 u-1}(\varphi)$ is not the whole space, we have $2 u+1-\operatorname{rk} E_{2}=\operatorname{rk} E_{2}^{c} \geq \operatorname{rk} E_{1}^{2}-1$ thus $\operatorname{rk}\left(E_{1}\right)+\operatorname{rk}\left(E_{2}\right) \leq 2 u+2$.

To exclude $\operatorname{rk}\left(E_{1}\right)+\operatorname{rk}\left(E_{2}\right)=2 u+2$, we observe that in such case, we have $D_{\left(\mathrm{rk}\left(E_{1}\right)-2\right)}\left(\varphi_{\left.E_{1}, E_{2}^{c}\right)}\right)$ is either empty or of codimension at most 2 . It cannot be of codimension 
at most 2 as it is contained in a Pfaffian variety, and hence, it must be empty. If it is empty, then $\varphi_{E_{1}, E_{2}^{c}}$ induces an embedding of vector bundles $E_{1}^{*}(-1) \rightarrow E_{2}^{c}$. This means that we have an exact sequence

$$
0 \rightarrow E_{1}^{*}(-1) \rightarrow E_{2}^{c} \rightarrow L \rightarrow 0
$$

where $L$ is a line bundle on $\mathbb{P}^{6}$. Now, since $E_{1}$ is a direct sum of line bundles and of twisted first differentials on $\mathbb{P}^{6}$, we have $\operatorname{Ext}_{\mathbb{P 6} 6}^{1}\left(L, E_{1}^{*}(-1)\right)=0$. We thus have $E_{2}^{c} \simeq E_{1}^{*}(-1) \oplus L$.

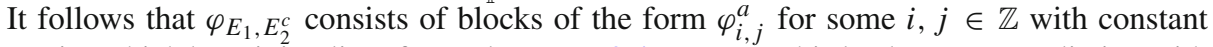
entries which by minimality of $E$ and Lemma 3.4 are zero. This leads to a contradiction with $\varphi_{E_{1}, E_{2}^{c}}$ being an embedding and proves that $\operatorname{rk}\left(E_{1}\right)+\operatorname{rk}\left(E_{2}\right) \neq 2 u+2$.

To exclude $\operatorname{rk}\left(E_{1}\right)+\operatorname{rk}\left(E_{2}\right)=2 u+1$, we shall prove that, in this case, we have $Y=$ $\operatorname{Pf}(\varphi) \cap D_{\left(\mathrm{rk}\left(E_{1}\right)-1\right)}\left(\varphi_{\left.E_{1}, E_{2}^{c}\right)}\right.$ is contained in $\operatorname{Pf}(\varphi)$ and is of codimension at most 2 in $\mathbb{P}^{6}$. To see the latter, we describe $Y$ as a degeneracy locus of a map between vector bundles of expected codimension 2. More precisely, we claim that $Y=D_{\left(\operatorname{rk}\left(E_{1}\right)-1\right)}(\kappa)$, where $\kappa=$ $\left(\kappa_{1}, \kappa_{2}\right): E_{1}^{*}(-1) \rightarrow\left(\operatorname{det} E_{3}\right)\left(u-\operatorname{rk} E_{1}\right) \oplus E_{2}^{c}$ with $\kappa_{2}=\varphi_{E_{1}, E_{2}^{c}}$ and

$$
\kappa_{1}=\varphi_{E_{1}, E_{3}} \wedge\left(\varphi_{E_{3}, E_{3}}\right)^{\wedge\left(u-\mathrm{rk}\left(E_{1}\right)\right)} \in H^{0}\left(\left(E_{1} \otimes \operatorname{det}\left(E_{3}\right)\right)\left(u+1-\mathrm{rk} E_{1}\right)\right) .
$$

Indeed, the claim follows directly from the following observation:

$$
\begin{aligned}
\varphi^{\wedge u}= & \left(\kappa_{1} \wedge\left(\varphi_{E_{1}, E_{2}}\right)^{\wedge\left(\mathrm{rk}\left(E_{1}\right)-1\right)},\left(\varphi_{E_{1}, E_{2}^{c}}\right)^{\wedge \mathrm{rk} E_{1}} \wedge \gamma\right) \\
& \in H^{0}\left(\left(\operatorname{det} E_{3} \otimes \operatorname{det}\left(E_{1}\right) \otimes \bigwedge^{r k E_{1}-1} E_{2}^{c}\right)(u)\right) \\
& \oplus H^{0}\left(\left(\operatorname{det}\left(E_{1}\right) \otimes \bigwedge^{\operatorname{rk} E_{3}-1} E_{3} \otimes \operatorname{det} E_{2}^{c}\right)(u)\right) \subset H^{0}\left(\bigwedge^{2 u} E(u)\right)
\end{aligned}
$$

for some $\gamma \in \bigwedge^{\mathrm{rk} E_{3}-1} E_{3}$.

Now, if $D_{\left(\mathrm{rk}\left(E_{1}\right)-1\right)}\left(\varphi_{\left.E_{1}, E_{2}^{c}\right)}\right.$ is nonempty, then it is a hypersurface in $\mathbb{P}^{6}$ and in consequence $Y=\operatorname{Pf}(\varphi) \cap D_{\left(\mathrm{rk}\left(E_{1}\right)-1\right)}\left(\varphi_{E_{1}, E_{2}^{c}}\right)$ is also nonempty. The codimension of $Y$ is then at most 2 giving a contradiction with $Y^{2} \subset \operatorname{Pf}(\varphi)$. If $D_{\left(\operatorname{rk}\left(E_{1}\right)-1\right)}\left(\varphi_{\left.E_{1}, E_{2}^{c}\right)}\right.$ is empty, we have $E_{2}^{c} \simeq$ $E_{1}^{*}(-1)$ and we get a contradiction with the minimality of $E$ as in the case $\operatorname{rk}\left(E_{1}\right)+\operatorname{rk}\left(E_{2}\right)=$ $2 u+2$.

Inequality 3.4, holding under the assumption that there exists an $i \in\left\{1 \ldots n_{\text {neg }}\right\}$ such that $i+1>n_{\text {pos }}$ or that $k_{i+1}+l_{i}<0$, together with Lemma 3.4 gives a contradiction with Lemma 3.5 and thus finishes the proof of Lemma 3.3.

Remark 3.6 Note that the quasi-Buchsbaum Calabi-Yau threefold of degree 15 can be described as the generic codimension 3 linear section of the Peskine Fano sixfold obtained as a degeneracy locus of a skew-symmetric morphism $T_{\mathbb{P}^{9}}(-2) \rightarrow \Omega_{\mathbb{P}^{9}}^{1}(1)$.

The following is a straightforward Corollary from Theorem 3.2.

Corollary 3.7 All quasi-Buchsbaum Calabi-Yau threefolds in $\mathbb{P}^{6}$ are arithmetically Buchsbaum. Moreover, arithmetically Cohen Macaulay Calabi-Yau threefolds in $\mathbb{P}^{6}$ are exactly those which correspond to case (1) in Theorem 3.2. 


\section{The degrees of Calabi-Yau threefolds in quadrics}

The approach to the classification of Calabi-Yau threefold in $\mathbb{P}^{6}$ by considering the minimal degrees of hypersurfaces that contain the Calabi-Yau threefolds is inspired by [21]. In this section, we shall work on quadrics of different ranks and dimensions. We shall use the following notation. For $k \leq 5$, we shall denote by $Q_{k}^{r} \subset \mathbb{P}^{k+1}$ a $k$-dimensional quadric hypersurface with corank $r$.

Proposition 4.1 Let $Q_{4}^{0} \subset \mathbb{P}^{5}$ be a smooth 4-dimensional quadric hypersurface. If $S$ is a nondegenerate canonically embedded surface of general type of degree d contained in $Q_{4}^{0}$, then $12 \leq d \leq 14$.

Proof Take $S$ a canonical surface of degree $d$ contained in a smooth 4-dimensional quadric $Q_{4}^{0}$. By the analog of the double-point formula for quadrics (see [19, Theorem 9.3]), the second Chern class of the normal bundle $c_{2}\left(N_{S \mid Q_{4}^{0}}\right)=S \cdot S$. Now, the Chow group $A^{2}\left(Q_{4}^{0}\right)$ of $Q_{4}^{0}$ has two generators corresponding to two families of planes on $Q_{4}^{0}$. We shall denote them $\theta_{1}$ and $\theta_{2}$. We have $\theta_{1}^{2}=\theta_{2}^{2}=1$ and $\theta_{1} \cdot \theta_{2}=0$. By definition, we know that $S \cdot\left(\theta_{1}+\theta_{2}\right)=d$, hence $S \sim a \theta_{1}+(d-a) \theta_{2}$ for some $a \in \mathbb{Z}$. It follows that

$$
c_{2}\left(N_{S \mid Q_{4}^{0}}\right)=2 a^{2}+d^{2}-2 d a .
$$

On the other hand, from the exact sequence

$$
\left.0 \rightarrow T_{S} \rightarrow T_{Q_{4}^{0}}\right|_{S} \rightarrow N_{S \mid Q_{4}^{0}} \rightarrow 0,
$$

we find that $c_{1}\left(N_{S \mid Q_{4}^{0}}\right)=5 h$ and

$$
c_{2}\left(N_{S \mid Q_{4}^{0}}\right)=12 h^{2}-c_{2}(S)=12 d-c_{2}(S),
$$

where $h=K_{S}$ is the restriction of the hyperplane class from $\mathbb{P}^{5}$ to $S$. It follows that

$$
c_{2}(S)=12 d-2 a^{2}-d^{2}+2 a d .
$$

On the other hand, using the Riemann-Roch formula, we have $7=\frac{1}{12}\left(d+c_{2}(S)\right)$, thus $c_{2}(S)=84-d$. By comparing both formulas, we infer

$$
84-d=12 d-2 a^{2}-d^{2}-2 a d .
$$

The only integers $d$ for which this equation has a solution are $d=12,13,14$.

Let us now consider a similar problem for a singular quadric.

Proposition 4.2 The degree d of a nondegenerate canonically embedded surface of general type $S \subset \mathbb{P}^{5}$ contained in a quadric cone $Q_{4}^{1} \subset \mathbb{P}^{5}$ is either 12 or 13 or 14 .

Proof Take $S$ a canonical surface of degree $d$ contained in a 4-dimensional quadric $Q_{4}^{1}$ of corank 1. Let us consider the projective bundle

$$
g: \mathfrak{F}:=\mathbb{P}\left(\mathcal{O}_{Q_{3}^{0}} \oplus \mathcal{O}_{Q_{3}^{0}}(1)\right) \rightarrow Q_{3}^{0},
$$

of the 1-dimensional quotients of the vector bundle $F:=\mathcal{O}_{Q_{3}^{0}} \oplus \mathcal{O}_{Q_{3}^{0}}(1)$. The linear system of its tautological line bundle $\mathcal{O}_{\mathfrak{F}}(1)$ defines a morphism $\pi: \mathfrak{F} \rightarrow Q_{4}^{1} \subset \mathbb{P}^{5}$. Observe that $\pi$ is the blow up of $Q_{4}^{1}$ in the singular point. Denote by $\xi$ and $h$ the pull back to $\mathfrak{F}$ by $\pi$ and 
$g$, respectively, of the hyperplane sections of $Q_{4}^{1}$ and $Q_{3}^{0}$. Let $S^{\prime}$ be the proper transform of $S \subset Q_{4}^{1}$ on $\mathfrak{F}$. By the Grothendieck relation, we have $\xi^{2}=h \xi$ so that $A^{2}(\mathfrak{F})$ is generated by $\xi^{2}$ and $h^{2}$. We can, hence, write the class of $S^{\prime}$ as $\left[S^{\prime}\right]=a \xi^{2}+b h^{2}$ where $a, b \in \mathbb{Q}$.

Observe that $S^{\prime}$ is smooth and either isomorphic to $S$ or the blow up of $S$ in the center of the cone $Q_{4}^{1}$ (when $S$ contains this center). From the double-point formula, we infer

$$
c_{2}\left(N_{S^{\prime} \mid \mathfrak{F}}\right)=\left(a \xi^{2}+b h^{2}\right)^{2}=\xi^{4}\left(a^{2}+2 a b\right)=2 a(d-a),
$$

since $d=\left[S^{\prime}\right] \xi^{2}=2 a+2 b$. Now, let us compute this Chern class using the exact sequence

$$
\left.0 \rightarrow T_{S^{\prime}} \rightarrow T_{\mathfrak{F}}\right|_{S^{\prime}} \rightarrow N_{S^{\prime} \mid \mathfrak{F}} \rightarrow 0 .
$$

We deduce that

$$
c_{2}\left(N_{S^{\prime} \mid \mathfrak{F}}\right)=c_{2}(\mathfrak{F})-c_{2}\left(S^{\prime}\right)-c_{1}\left(S^{\prime}\right) c_{1}\left(N_{S^{\prime} \mid \mathfrak{P}}\right)
$$

Next, we use,

$$
\begin{aligned}
& 0 \rightarrow \mathcal{O}_{\mathfrak{F}} \rightarrow g^{*} F^{*}(1) \rightarrow T_{\mathfrak{F} \mid Q_{3}^{0}} \rightarrow 0 \\
& 0 \rightarrow T_{\mathfrak{F} \mid Q_{3}^{0}} \rightarrow T_{\mathfrak{F}} \rightarrow g^{*} T_{Q_{3}^{0}} \rightarrow 0 .
\end{aligned}
$$

We obtain $c_{2}\left(N_{S^{\prime} \mid \mathfrak{F}}\right)=12 d-84+2 a$ using the fact that $c_{1}\left(S^{\prime}\right)=\left.(-2 \xi+h)\right|_{S^{\prime}}$ and the Noether formula $c_{2}\left(S^{\prime}\right)=84-h^{2}\left[S^{\prime}\right]=84-2 a$. We infer the equation:

$$
a^{2}+a(1-d)+6 d-42=(a-6)(a-d+7)=0 .
$$

We thus deduce that either $a=6$ and $b=\frac{d}{2}-6$ or $a=d-7$ and $b=7-\frac{1}{2} d$.

Now, the exceptional divisor $\Xi \subset \mathfrak{F}$ of $\pi$ is in the linear system $|\xi-h|$. Since $S$ is smooth, we have one of the followings:

- either $\left.\Xi\right|_{S^{\prime}}=0$ and $S^{\prime}$ is isomorphic to $S$;

- or $\left(\left.\Xi\right|_{S^{\prime}}\right)^{2}=-1$ and $S^{\prime}$ is the blow up of $S$ in one point.

In the first case, we deduce that $b=0$ so either $d=12$ or $d=14$. In the second case, $b=\frac{1}{2}$ so $d=13$.

Theorem 4.3 The degree $d$ of a nondegenerate Calabi-Yau threefold $X$ embedded in a quadric in $\mathbb{P}^{6}$ of corank $\leq 2$ is bounded by $12 \leq d \leq 14$.

Moreover, the degree of a nondegenerate Calabi-Yau threefold contained in a smooth quadric cannot be 13 .

Proof First, remark that a generic hyperplane section of $X \subset \mathbb{P}^{6}$ is a canonically embedded surface of general type contained either in a smooth quadric $Q_{4}^{0}$ or in a corank 1 quadric $Q_{4}^{1} \subset \mathbb{P}^{5}$. We obtain the bound on the degree from Propositions 4.2 and 4.1.

To prove the second part, observe that $A^{2}\left(Q_{5}^{0}\right)$ has only one generator $\theta$ and that the restriction $\left.\theta\right|_{H}$ to a hyperplane section $H=Q_{4}^{0}$ is $\theta_{1}+\theta_{2}$ in the notation of Proposition 4.2. It follows that, in this case, in the proof of Proposition 4.1, we may additionally assume $a=b$. It is now enough to observe that the Eq. (4.1) has no solution for $d=13$ giving $a=b$. In fact, when $d=13$, we must have $S \sim 6 \theta_{1}+7 \theta_{2}$.

To conclude the proof of Theorem 1.2, it is enough to prove the following:

Proposition 4.4 If $X$ is a smooth Calabi-Yau threefolds contained in a quadric $Q_{5}^{r}$ of corank $r \geq 3$ in $\mathbb{P}^{6}$, then $X$ is contained in a linear space contained in $Q_{5}^{r}$. 
Proof The proof will consist of two steps which will be formulated as lemmas.

Lemma 4.5 Let $X$ be a smooth threefold contained in a quadric $Q_{5}^{r}$ of corank $r \geq 3$ in $\mathbb{P}^{6}$. Then one of the followings holds:

(1) $X$ is contained in a linear space $L \subset Q_{5}^{r}$

(2) $X$ is fibered by surfaces contained in linear spaces contained in $Q_{5}^{r}$.

Proof If the quadric $Q_{5}^{r}$ is of corank $\geq 5$, then it decomposes into hyperplane and $X$ being smooth must be contained in one of them.

If the quadric $Q_{5}^{r}$ has corank 4, then it is a cone $Q_{5}^{4}$ with center some $\mathbf{P} \simeq \mathbb{P}^{3}$ and spanned over a smooth conic $Q_{1}^{0}$. Consider the hyperplane $H_{l}$ spanned by $\mathbf{P}$ and any line in the plane spanned by $Q_{1}^{0}$. Observe that $H_{l} \cap Q$ decompose as a sum of two linear spaces $L_{l}^{1}$ and $L_{l}^{2}$. It follows that $H_{l} \cap X$ is either the whole $X$ for some $l$ or is a divisor on $X$ decomposed as $S_{L}=S_{l}^{1}+S_{l}^{2}$ with $S_{l}^{1} \subset L_{l}^{1}$ and $S_{l}^{2} \subset L_{l}^{2}$. In the first case, the assertion follows as $H_{l} \cap Q_{5}^{4}$ is a union of two linear spaces and $X$ is irreducible. We, hence, need only to consider $X$ having a 1-dimensional linear system given by surfaces $S_{l}^{i}$ which are clearly all in the same system independently on $i \in\{1,2\}$. Take two distinct points $q, q^{\prime} \in Q_{1}^{0}$. For a 1-dimensional linear system on a smooth threefold, we have two possibilities:

- the system is base point free, in which case, we have a fibration of $X$ given by surfaces $S_{q}$ giving the assertion,

- or the system has a base curve $C$.

In the latter case, we claim that $\mathbf{P}$ must be the space tangent to $X$ in every point of this curve. Indeed, all hyperplanes containing $\mathbf{P}$ intersect $X$ in the union of two surfaces passing through $C$, and hence, the intersection is singular in every point of $C$. It follows that the tangent space to $X$ in any of the points of $C$ is contained in $\mathbf{P}$ thus is equal to $\mathbf{P}$. We now use the famous Zak theorem on tangencies, which for smooth varieties is formulated as follows.

Theorem 4.6 (Zak [38]). Let $X^{n} \subset \mathbb{P}^{n+a}$ be a subvariety not contained in a hyperplane. Fix any linear space $L=\mathbb{P}^{n+k} \subset \mathbb{P}^{n+a}, 0 \leq k \leq a-1$. Then

$$
\operatorname{dim}\left\{x \in X \mid \tilde{T}_{x} X \subset L\right\} \leq k,
$$

where $\tilde{T}_{X} X$ denotes the embedded tangent space to $X$ in $x$.

Zak's theorem implies that a tangent projective space to a threefold in $\mathbb{P}^{6}$ cannot be tangent to it in a curve. In particular, $\mathbf{P}$ cannot be tangent to $X$ in $C$. The contradiction concludes the proof for $r=4$. Let us point out that from now on, we shall consider $\tilde{T}_{X} X$ as standard notation for the embedded tangent space.

Finally, assume that $r=3$, i.e., the quadric $Q_{5}^{r}$ is of rank 4. Then $Q_{5}^{3}$ is a cone with center a plane $\mathbf{P}$ and spanned over a smooth quadric $Q_{2}^{0}$ of dimension 2. Consider the family $\left\{X_{q}\right\}_{q \in Q_{2}^{0}}$ consisting of intersections of $X$ with hyperplanes $H_{q}$ spanned by $\mathbf{P}$ and planes $\tilde{T}_{q} Q_{2}^{0}$ tangent to $Q_{2}^{0}$ parametrized by the tangency points. Observe that $\tilde{T}_{q} Q_{2}^{0} \cap Q_{2}^{0}$ consists of two lines $l_{1}^{q}$ and $l_{2}^{q}$ with $q=l_{1}^{q} \cap l_{2}^{q}$, hence, $H_{q} \cap Q_{5}^{3}$ is the union of two 4-dimensional linear spaces $L_{1}^{q}$ and $L_{2}^{q}$ spanned by $\mathbf{P}$ and lines $l_{1}^{q}$ and $l_{2}^{q}$, respectively. If $X_{q}=X$ for some $q \in Q_{2}^{0}$ then $X$, being irreducible, is contained in one of the two linear spaces $L_{i}^{p}$ for $i=1$ or 2 and the assertion follows. If $X_{q} \neq X$ for all $q \in Q_{2}^{0}$ then $X_{q}$ is decomposed as $X_{q}=S_{1}^{q} \cup S_{2}^{q}$. We thus have two 1-dimensional linear systems on $X$ and as in the previous case, we have the following possibilities 
- one of the linear systems is base point free giving us a fibration by surfaces as in the assertion

- both systems contain a base curve.

In the latter case, let us denote the base curves by $C_{1}$ and $C_{2}$. Then $C_{1} \cap C_{2} \neq \varnothing$ as these are plane curves. Let us choose any point $p \in C_{1} \cap C_{2}$. Now, for every $q$, we have $H_{q} \cap X=S_{1}^{q} \cup S_{2}^{q}$ is a reducible surface which is clearly singular in $p \in C_{1} \cap C_{2} \subset S_{1}^{q} \cap S_{2}^{q}$. It follows that $\tilde{T}_{p} X \subset H_{q}$ for all $q \in Q_{2}^{0}$. Thus, $\tilde{T}_{p} X \subset \bigcap_{q \in Q_{5}^{3}} H_{q}=\mathbf{P}$. As $\tilde{T}_{p} X$ is of dimension 3 and $\mathbf{P}$ is a plane, we get a contradiction excluding the last case and finishing the proof.

Lemma 4.7 There are no smooth Calabi-Yau threefolds contained in a quadric $Q_{5}^{r}$ of corank $3 \leq r \leq 4$ which admit a fibration by surfaces contained in linear spaces contained in $Q_{5}^{r}$.

Proof Assume the contrary. Let $X$ be a smooth Calabi-Yau threefold contained in $Q_{5}^{r}$ such that $X$ admits a fibration $f: X \rightarrow Y$ with fibers being surfaces contained in linear spaces contained in $Q_{5}^{r}$. As usual, we use the notation for which $Q_{5}^{r}$ will be a cone with center a linear space $\mathbf{P}$ and spanned over a smooth quadric $Q_{5-r}^{0}$ of dimension $5-r$. As $X$ is a Calabi-Yau threefold, we have $Y \simeq \mathbb{P}^{1}$ and the fiber $X_{y}$ for a generic point $y \in Y$ is either a K3 or an abelian surface (smooth in both cases by Bertini theorem). Observe moreover that the maximal dimensional linear spaces in $Q_{5}^{r}$ are projective spaces of dimension 4 . In both cases, we have only 1-dimensional families of $\mathbb{P}^{4}$ contained in $Q_{5}^{r}$ and no two fibers of the fibration can be contained in the same $\mathbb{P}^{4}$ as otherwise they would have to intersect. It follows that we can choose a family $\left\{L_{y}\right\}_{y \in Y}$ such that $L_{y} \simeq \mathbb{P}^{4} \subset Q_{5}^{r}$ and $L_{y} \cap L_{y}^{\prime}=\mathbf{P}$ for any distinct $y, y^{\prime} \in Y$ such that for a generic $y \in Y$ the fiber $X_{y}$ is either a K3 or an abelian surface contained in $L_{y}$.

Claim We claim that such a fiber $X_{y}$ must be one of the followings:

- a complete intersection of a quadric and a cubic in $L_{y} \simeq \mathbb{P}^{4}$

- a complete intersection of a hyperplane and a quartic in $L_{y} \simeq \mathbb{P}^{4}$

- the zero locus of the Horrocks-Mumford bundle on $L_{y} \simeq \mathbb{P}^{4}$

Indeed, fix a generic $y \in Y$. The fiber $X_{y}$ is then a smooth $\mathrm{K} 3$ or abelian surface contained in $L_{y}=\mathbb{P}^{4}$. The theorem of Severi, saying that the only surface in $\mathbb{P}^{4}$ which is not linearly normal is the Veronese surface, implies that $X_{y}$ is linearly normal. Let us denote its degree by $d_{X}$. The following computation applies:

By the double-point formula, $c_{2}\left(N_{X_{y} \mid \mathbb{P}^{4}}\right)=\left[X_{y}\right]^{2}=d_{X_{y}}^{2}$. On the other hand, by the exact sequence:

$$
\left.0 \rightarrow T_{X_{y}} \rightarrow T_{\mathbb{P}^{4}}\right|_{S} \rightarrow N_{X_{y} \mid \mathbb{P}^{4}} \rightarrow 0
$$

and the vanishing $c_{1}\left(T_{X_{y}}\right)=0$, we have:

$$
c_{2}\left(N_{X_{y} \mid \mathbb{P}^{4}}\right)=c_{2}\left(\left.T_{\mathbb{P}^{4}}\right|_{X_{y}}\right)-c_{2}\left(T_{X_{y}}\right)= \begin{cases}10 d_{X_{y}}-24 & \text { when } X_{y} \text { is a K3 surface } \\ 10 d_{X_{y}} & \text { when } X_{y} \text { is an abelian surface }\end{cases}
$$

Comparing the two equations, we have $d_{X_{y}}=4$ or 6 and $X_{y}$ is a K3 surface or $d_{X_{y}}=10$ and $X_{y}$ is an abelian surface. Finally, we use the classification (cf. [12, Table 7.3]) of non-general type surfaces in $\mathbb{P}^{4}$ with $d_{X_{y}}=4,6,10$ to obtain our claim.

From now on, we shall consider the cases of rank $r=3$ and $r=4$ separately. For $r=3$, we have $\mathbf{P}$ is a 3 -dimensional space and $Q_{5}^{4}$ is a cone centered at $\mathbf{P}$ and spanned over a 
conic $Q_{1}^{0}$. A generic fiber $X_{y}$ meets $\mathbf{P}$ in a curve $C_{y}$, and all these curves must be disjoint. It follows that $X \cap \mathbf{P}$ contains a surface $\mathbf{S}$ fibered by the curves $C_{y}$. Observe, moreover, that since the fibers $X_{y}$ cover $X$, it follows that $\mathbf{S}=X \cap \mathbf{P}$ is of pure dimension 2. Now, by the Zak tangencies theorem, $\mathbf{S}$ is smooth in codimension 1 (since any singular point of $\mathbf{S}$ is a tangency point of $\mathbf{P}$ with $X$ ) and since it is a hypersurface it must be irreducible with isolated singularities. Observe now that it follows that the hyperplane class $H$ on $X$ is linearly equivalent to $\mathbf{S}+2 X_{y}$. We shall compute the degree of $\mathbf{S}$ in terms of the degrees of the fiber $X_{y}$ in two ways. The first will be based on the adjunction formula on $\mathbf{S}$. Since $C_{y}$ is smooth, we can compute its canonical class:

- On one hand, $C_{y}$ is a hyperplane section of $X_{y}$ hence a canonical curve of degree $d=4,6$ or 10 hence

$$
\operatorname{deg}\left(K_{C_{y}}\right)=\operatorname{deg}\left(X_{y}\right) .
$$

- On the other $C_{y}$ is a fiber of a fibration on the hypersurface $\mathbf{S} \subset \mathbf{P}=\mathbb{P}^{3}$ hence $\operatorname{deg}\left(K_{C_{y}}\right)=$ $\operatorname{deg}(\mathbf{S})-4$.

Next, by adjunction on $X$, we have $\omega_{\mathbf{S}}=\left.\mathbf{S}\right|_{\mathbf{S}}=\left.\left(H-2 X_{y}\right)\right|_{\mathbf{S}}=\left.H\right|_{\mathbf{S}}-2 C_{y}$. But, on the other hand, on $\mathbf{P}$ we have $\omega_{\mathbf{S}}=\left.(\operatorname{deg}(\mathbf{S})-4) H\right|_{\mathbf{S}}$. It follows that $\operatorname{deg}\left(C_{y}\right)=\frac{1}{2}(5-$ $\operatorname{deg}(\mathbf{S})) \operatorname{deg}(\mathbf{S}))$ and, by comparing with $\operatorname{deg}\left(C_{y}\right)=\operatorname{deg}\left(K_{C_{y}}\right)=\operatorname{deg}(\mathbf{S})-4$, we get a contradiction with $\operatorname{deg}(\mathbf{S})$ being an integer. This concludes the proof for $r=3$.

Let us pass to the case $r=4$. We then have $\mathbf{P}$ is a plane and $Q_{5}^{3}$ is a cone centered in $\mathbf{P}$ and spanned over a quadric surface $Q_{2}^{0}$. In this case, the generic fiber $X_{y}$ meets $\mathbf{P}$ in a finite set of points. It follows that $X \cap \mathbf{P}$ is a curve $\mathbf{C}$. Consider for a generic $p \in Q_{2}^{0}$ the hyperplane $H_{p}$ spanned by $\mathbf{P}$ and the tangent $\tilde{T}_{p} Q_{2}^{0}$. The intersection $H_{p} \cap Q_{5}^{3}$ decomposes into two 3-dimensional linear spaces $L_{1}$ and $L_{2}$ such that $L_{1}$ contains a fiber $S$. It follows that $H_{p} \cap X$ decomposes into a fiber $X_{y}$ of the fibration and a surface $T$ such that $T$ contains $\mathbf{C}$ and $T=X \cap L_{2}$. Denote the blow up of $Q_{5}^{3}$ in $L_{2}$ by $\pi: \mathfrak{P} \rightarrow Q_{5}^{3}$ and the proper transform of $X$ by the blow up by $\tilde{X}$. Since $X \cap L_{2}$ is a Cartier divisor it follows that $\pi: \tilde{X} \rightarrow X$ is an isomorphism. Our aim is to prove that this is in fact impossible and in this way finish the proof. We achieve our aim by performing a computation on the resolution $\mathfrak{P}$ proving that $\tilde{X}$ must contain a fiber of the projection $\pi$. The computation is presented below.

First, observe that $\mathfrak{P} \simeq \mathbb{P}\left(2 \mathcal{O}_{\mathbb{P}^{1}}(1) \oplus 3 \mathcal{O}_{\mathbb{P}^{1}}\right)$ admits a natural fibration $g: \mathfrak{P} \rightarrow \mathbb{P}^{1}$. The Chow group of $\mathfrak{P}$ is generated by the pullback $\xi=\pi^{*}(H)$ of the hyperplane section $H$ of $Q_{5}^{3}$ and the fiber $h=g^{*}([p t])$ with the relation $\xi^{5}=2 \xi^{4} \cdot h$ and $h^{2}=0$. It follows that the class of $X$ has a decomposition $[X]=d_{X_{y}} \xi^{2}+\left(d-2 d_{X_{y}}\right) h \xi$.

Claim We claim that one of the followings holds:

- $d_{X_{y}}=6$ and $[\tilde{X}]=6 \xi^{2}+(d-12) h \xi$

- $[\tilde{X}]=10 \xi^{2}-3 h \xi$

- $[\tilde{X}]=4 \xi^{2}+3 h \xi$

The claim follows from a computation with the double-point formula analogous to the proof of Theorem 4.2. Indeed, consider a section of $X$ by a generic element from the system $\xi$. It is a surface of general type $G$ embedded in a variety $\mathfrak{P}^{\prime} \simeq \mathbb{P}\left(2 \mathcal{O}_{\mathbb{P}^{1}}(1) \oplus 2 \mathcal{O}_{\mathbb{P}^{1}}\right)$ with generators of the Chow group $\xi^{\prime}=\left.\xi\right|_{\mathfrak{P}^{\prime}}$ and $h^{\prime}=\left.h\right|_{\mathfrak{P}^{\prime}}$ such that $[G]=d_{X_{y}} \xi^{\prime 2}+(d-$ $\left.2 d_{X_{y}}\right) h^{\prime} \xi^{\prime}$. By the double-point formula, we have $c_{2}\left(N_{G \mid \mathfrak{P}^{\prime}}\right)=[G]^{2}=2 d d_{X_{y}}-2 d_{X_{y}}^{2}$. On the other hand, by the exact sequence

$$
0 \rightarrow T_{G} \rightarrow T_{\mathfrak{P}^{\prime}} \rightarrow N_{G \mid \mathfrak{P}^{\prime}} \rightarrow 0
$$


and the Riemann-Roch theorem on $G$, we obtain another formula $c_{2}\left(N_{G \mid \mathfrak{P}^{\prime}}\right)=2 d_{X_{y}}+$ $12 d-84$. Comparing the two we prove the claim.

Before we go further, let us recall the following.

Theorem 4.8 (Serre's construction (for this version see [1, Theorem 1.1])). Let $X$ be a smooth variety of dimension $\geq 3$ and $L$ a line bundle on $X$ such that $h^{2}\left(L^{-1}\right)=0$. Let $Y$ be a locally complete intersection subscheme of pure codimension 2. Then $Y \subset X$ is the zero locus of a section of a rank two vector bundle $\mathcal{E}$ on $X$ if and only if $\omega_{Y}=\left.\left(\omega_{X} \otimes L\right)\right|_{Y}$. Moreover in such a case, we have the following exact sequence

$$
0 \rightarrow \mathcal{O}_{X} \rightarrow \mathcal{E} \rightarrow \mathcal{I}_{Y} \otimes L \rightarrow 0 \text {. }
$$

By the Serre's construction, $\tilde{X}$ is obtained as the zero locus of a section of a rank 2 vector bundle $\mathcal{E}$ on $\mathfrak{P}$. We have $c_{1}(\mathcal{E})=5 \xi$ and $c_{2}(\mathcal{E})=[\tilde{X}]$. Consider first the case $d_{S}=6$. We see that the restriction of $\mathcal{E}$ to any fiber $F_{t}$ of $g$ decomposes as $\mathcal{O}_{F_{t}}(2) \oplus \mathcal{O}_{F_{t}}(3)$. Indeed, the zero locus of the section defining $X$ restricted to $F_{t}$ is Gorenstein of codimension 2 as a divisor on $X$, hence, is a complete intersection. This means that the restricted bundle decomposes over every fiber and this decomposition is the same as for the generic fiber, i.e., $\left.\mathcal{E}\right|_{F_{t}}=\mathcal{O}_{F_{t}}(2) \oplus \mathcal{O}_{F_{t}}(3)$.

It follows that $g_{*} \mathcal{E}(-3 \xi)$ is a line bundle and that $R^{i} g_{*} \mathcal{E}(-3 \xi)=0$ for $i=1,2$. This implies that $\chi\left(g_{*} \mathcal{E}(-3 \xi)\right)=\chi(\mathcal{E}(-3 \xi))$. The latter is computed by the HirzebruchRiemann-Roch theorem on $\mathfrak{P}$ to be $13-d$.

Hence, $g_{*} \mathcal{E}(-3 \xi)=\mathcal{O}_{\mathbb{P}^{1}}(12-d)$. Now, by the projection formula, we have $\mathcal{E}(-3 \xi+(d-$ $12) h)$ has a section. Since $c_{2}(\mathcal{E}(-3 \xi+(d-12) h))=0$ and $\mathcal{E}(-3 \xi+(d-12) h) \otimes \mathcal{O}(-D)$ has no section for nontrivial effective divisors $D$, the section does not vanish. It follows that we have the following exact sequence:

$$
0 \rightarrow \mathcal{O}_{\mathfrak{P}}(3 \xi-(d-12) h) \rightarrow \mathcal{E} \rightarrow \mathcal{O}_{\mathfrak{P}}(2 \xi+(d-12) h)
$$

Let us now restrict $\mathcal{E}$ to the exceptional locus $\Xi \simeq \mathbb{P}^{2} \times \mathbb{P}^{1}$ of the blow up $\pi$ and denote $\pi^{\prime \prime}=\left.\pi\right|_{\Xi}$ the projection onto $\mathbb{P}^{2}$. We observe that under the assumption that $\tilde{X}$ contains no fiber of the projection $\pi$, we have $\mathcal{E}^{\prime}=\left.\mathcal{E}\right|_{E}$ restricted to every fiber of the projection $\pi^{\prime \prime}$ is a bundle of rank 2 with trivial first Chern class and either a nonvanishing section or a section vanishing in one point, i.e., it is either $\mathcal{O}_{\mathbb{P}^{1}} \oplus \mathcal{O}_{\mathbb{P}^{1}}$ or $\mathcal{O}_{\mathbb{P}^{1}}(-1) \oplus \mathcal{O}_{\mathbb{P}^{1}}$ (1). It follows that $\pi_{*}^{\prime \prime}\left(\mathcal{E}^{\prime}\right)$ is a vector bundle of rank 2 and $R^{i} \pi_{*}^{\prime \prime} \mathcal{E}^{\prime}=0$. We compute $c_{2}\left(\pi_{*}^{\prime \prime}\left(\mathcal{E}^{\prime}\right)\right)$. By restricting the exact sequence 4.2 to $\Xi$, we get

$$
0 \rightarrow \mathcal{O}_{E}\left(3 \xi^{\prime}-(d-12) h^{\prime}\right) \rightarrow \mathcal{E}^{\prime} \rightarrow \mathcal{O}_{E}\left(2 \xi^{\prime}+(d-12) h^{\prime}\right) .
$$

Taking the push-forward by $\pi^{\prime \prime}$, we have:

$$
\begin{aligned}
0 \rightarrow & \pi_{*}^{\prime \prime}\left(\mathcal{O}_{E}\left(3 \xi^{\prime}-(d-12) h^{\prime}\right)\right) \rightarrow \pi_{*}^{\prime \prime} \mathcal{E}^{\prime} \rightarrow \pi_{*}^{\prime \prime} \mathcal{O}_{E}\left(2 \xi^{\prime}+(d-12) h^{\prime}\right) \\
& \rightarrow R^{1} \pi_{*}^{\prime \prime}\left(\mathcal{O}_{E}\left(3 \xi^{\prime}-(d-12) h^{\prime}\right)\right) \rightarrow R^{1} \pi_{*}^{\prime \prime} \mathcal{E}^{\prime} \ldots
\end{aligned}
$$

We now observe that $\pi_{*}^{\prime \prime}\left(\mathcal{O}_{E}\left(3 \xi^{\prime}-(d-12) h^{\prime}\right)\right)=0$ as $\mathcal{O}_{E}\left(3 \xi^{\prime}-(d-12) h^{\prime}\right)$ restricted to any fiber of $\pi$ has no nontrivial section. Moreover, $R^{1} \pi_{*}^{\prime \prime} \mathcal{E}^{\prime}=0$ as $\mathcal{E}^{\prime}$ restricted to any fiber of $\pi$ is either $2 \mathcal{O}_{\mathbb{P}^{1}}$ or $\mathcal{O}_{\mathbb{P}^{1}}(-1) \oplus \mathcal{O}_{\mathbb{P}^{1}}$ (1). Finally, by projection formula and base change

$$
\pi_{*}^{\prime \prime} \mathcal{O}_{\Xi}\left(2 \xi^{\prime}+(d-12) h^{\prime}\right)=\mathcal{O}_{\mathbb{P}^{2}}(2) \otimes H^{0}\left(\mathbb{P}^{1}, \mathcal{O}_{\mathbb{P}^{1}}(d-12)\right)=(d-11) \mathcal{O}_{\mathbb{P}^{2}}(2)
$$

and

$$
\begin{aligned}
& R^{1} \pi_{*}^{\prime \prime}\left(\mathcal{O}_{\Xi}\left(3 \xi^{\prime}-(d-12) h^{\prime}\right)\right)=\mathcal{O}_{\mathbb{P}^{2}}(3) \otimes H^{1}\left(\mathbb{P}^{1}, \mathcal{O}_{\mathbb{P}^{1}}(12-d)\right) \\
& =\mathcal{O}_{\mathbb{P}^{2}}(3) \otimes H^{0}\left(\mathbb{P}^{1}, \mathcal{O}_{\mathbb{P}^{1}}(d-14)\right)=(d-13) \mathcal{O}_{\mathbb{P}^{2}}(3),
\end{aligned}
$$


and we get

$$
0 \rightarrow \pi_{*}^{\prime \prime} \mathcal{E}^{\prime} \rightarrow(d-11) \mathcal{O}_{\mathbb{P}^{2}}(2) \rightarrow(d-13) \mathcal{O}_{\mathbb{P}^{2}}(3) \rightarrow 0 .
$$

From the exact sequence, we compute the second Chern class of the rank 2 bundle $\pi_{*}^{\prime \prime} \mathcal{E}^{\prime}$ :

$$
c_{2}\left(\pi_{*}^{\prime \prime} \mathcal{E}^{\prime}\right)=\frac{1}{2} d^{2}-\frac{29}{2} d+108
$$

The latter is nonzero for $d \in \mathbb{Z}$. This means that every section of $\pi_{*}^{\prime \prime}\left(\mathcal{E}^{\prime}\right)$ vanishes in some point on $\mathbb{P}^{2}$, and hence, every section of $\mathcal{E}^{\prime}$ vanishes on some fiber of $\pi^{\prime \prime}$. Finally, this implies that $\tilde{X}$ contains a fiber of the blow up $\pi$ giving a contradiction which completes the proof in the case $[\tilde{X}]=6 \xi^{2}+(d-12) h \xi$.

The proof in the case $[\tilde{X}]=4 \xi^{2}+3 \xi \cdot h$ is completely analogous leading to $c_{2}\left(\pi_{*}^{\prime \prime}\left(\mathcal{E}^{\prime}\right)\right)=$ 1. The case $[\tilde{X}]=10 \xi^{2}-3 \xi \cdot h$ is excluded because it has negative intersection with $[\Xi] \xi=\left(\xi^{2}-2 \xi h\right) \xi=\xi^{3}-2 \xi^{2} h$.

We have proved in Theorem 1.2 that there are no smooth Calabi-Yau threefolds of degrees 11 and 15 contained in a quadric. For canonical surfaces of general type, the same bounds apply only if we restrict the rank of the quadric to be at least 5. In fact, the proof of Lemma 4.7 suggests that one may construct nodal Calabi-Yau threefolds contained in quadrics of rank 4.

Below, we recall examples of nodal Calabi-Yau threefolds and in consequence also smooth canonical surfaces of general type of degrees 11 and 15 contained in quadrics of rank 4 .

Proposition 4.9 Let $E_{11}=3 \mathcal{O}_{\mathbb{P}^{6}}(1) \oplus 2 \mathcal{O}_{\mathbb{P}^{6}}(-1)$ and let $\sigma_{11} \in H^{0}\left(\left(\bigwedge^{2} E_{11}\right)(1)\right)$ be a general section. Then $X_{11}=\operatorname{Pf}\left(\sigma_{11}\right)$ is well defined and is a singular Calabi-Yau threefold with singular locus consisting of one ordinary double point.

Proof Since $E_{11}$ is a decomposable bundle, the variety $X_{11}$ is scheme theoretically defined by Pfaffians of a skew-symmetric matrix with entries being general polynomials of degrees of the shape:

$$
\left(\begin{array}{cccc}
3 & 3 & 1 & 1 \\
& 3 & 1 & 1 \\
& & 1 & 1 \\
& & & -
\end{array}\right)
$$

where - corresponds to the zero entry. It is hence contained in a 2-dimensional system of quadrics defining a cone over $\mathbb{P}^{1} \times \mathbb{P}^{2}$. This system of quadrics is generated by the $2 \times 2$ minors of a $3 \times 2$ matrix of linear forms. It follows that all quadrics in the system are of rank at most 4. Let us choose a general quadric in the system and denote it $Q_{5}^{3}$. Then $Q_{5}^{3}$ is a cone with vertex a plane $\mathbf{P}$ and spanned over a smooth quadric surface $Q_{3}^{0}$. The quadric $Q_{3}^{0}$ has two fibrations giving two systems of Weil divisors on $X_{11}$. The generic element of one of them is a complete intersection of a hyperplane and a quartic obtained as a Pfaffian of the form

$$
\left(\begin{array}{lll}
3 & 3 & 1 \\
& 3 & 1 \\
& & 1
\end{array}\right)
$$

the generic element of the other is a surface of degree 7 given by $2 \times 2$ minors of a general matrix of the form

$$
\left(\begin{array}{lll}
3 & 1 & 1 \\
3 & 1 & 1
\end{array}\right)
$$


It follows now from the end of proof of Lemma 4.7 that in the notation of this proof, we have $[\tilde{X}]=4 \xi^{2}+3 \xi \cdot h$ leading to $c_{2}\left(\pi_{*}^{\prime \prime}\left(\mathcal{E}^{\prime}\right)\right)=1$. Hence, $X_{11}$ contains a singular locus of degree at least 1 . An example with one node is given by a Macaulay2 ([20]) calculation.

Remark 4.10 The singular point of $X_{11}$ can be found as the intersection of zeros of the linear entries of the skew-symmetric matrix defining it.

Let $\phi: 10 \mathcal{O}_{\mathbb{P} 6} \rightarrow 2 \mathcal{O}_{\mathbb{P} 6}(1)$ be a general map. Let $E_{15}=\operatorname{ker}(\phi) \oplus \mathcal{O}(1)$. Let $\sigma_{15} \in$ $H^{0}\left(\left(\bigwedge^{2} E_{15}\right)(1)\right)$ be a generic section.

Proposition 4.11 Under above assumptions, the variety $B_{15}=\operatorname{Pf}\left(\sigma_{15}\right)$ is well defined and is a singular Calabi-Yau threefold with singular locus consisting of three ordinary double points.

Proof Similarly as in the proof for $d=11$, we prove that $c_{2}\left(\pi_{*}^{\prime \prime}\left(\mathcal{E}^{\prime}\right)\right)=3$ hence $B_{15}$ contains a singular locus of degree at least 3 . The bound 3 is reached by Macaulay2 ([20]) calculation.

Remark 4.12 We saw that both Calabi-Yau threefolds $X_{11}$ and $B_{15}$ admit two birational smooth resolution of Picard rank 2. The extremal rays of these resolutions consist of the small contraction of lines and one K3 fibration and one elliptic fibration. This means that the two birational models give all Calabi-Yau birational models of these varieties.

\section{Classification of Calabi-Yau threefolds contained in five-dimensional quadrics}

In this section, we classify all nondegenerate Calabi-Yau threefolds contained in 5dimensional quadrics. Let $X$ be such a Calabi-Yau threefold. We know from previous sections that $12 \leq \operatorname{deg}(X) \leq 14$. Moreover, from the Riemann Roch theorem, we know that any nondegenerate Calabi-Yau threefold of degree at most 13 in $\mathbb{P}^{6}$ is contained in a quadric, and hence, our classification contains all Calabi-Yau threefolds contained in $\mathbb{P}^{6}$ of degree at most 13.

Corollary 5.1 A Calabi-Yau threefold $X \subset \mathbb{P}^{6}$ has degree $d \geq 12$. Moreover, the degree 12 threefold is a complete intersection of two quadrics and a cubic and the degree 13 threefold is given by the $4 \times 4$ Pfaffians of a $5 \times 5$ skew-symmetric matrix with linear entries except one row and one column of quadrics.

Proof We deduce from the Riemann-Roch theorem that

$$
\chi\left(\mathcal{O}_{X}(m)\right)=\frac{1}{6} m d\left(m^{2}-1\right)+7 m .
$$

Thus, by Serre duality and Kodaira vanishing for $d \leq 13$, we have $h^{0}\left(\mathcal{O}_{X}(2)\right) \leq \chi\left(\mathcal{O}_{X}(2)\right) \leq$ $27<28=h^{0}\left(\mathcal{O}_{\mathbb{P}^{6}}(2)\right)$. It follows that $X$ has to be contained in a quadric so the first part follows from Theorem 1.2. In the case $\operatorname{deg} X=12$, the threefold $X$ is contained in a pencil of quadrics. From Proposition 4.4, all the quadrics containing $X$ have rank $\geq 5$ and from the proof of Proposition 4.2, their singular locus is always disjoint from $X$. In particular, a general quadric containing $X$ is smooth (by the Bertini theorem) and the intersection $\mathcal{Q}$ of two quadrics containing $X$ is smooth along $X$. We now apply the Lefschetz hyperplane theorem to deduce that the Picard group of $\mathcal{Q}$ is generated by the hyperplane section from $\mathbb{P}^{6}$. Since $X$ does not pass through the singular locus of $\mathcal{Q}$, it is a Cartier divisor, and hence, $X$ is cut out by a hypersurface in $\mathbb{P}^{6}$ of degree three. It follows that $X$ is a complete intersection of two quadrics and a cubic. 
In the case $d=13$, we deduce from Theorem 4.3 that $X$ is not contained in a smooth quadric. Moreover, from the proof of Theorem 4.3, the singular locus of a quadric containing $X$ is contained in $X$. We know also from Theorem 1.2 that the ranks of the quadrics containing $X$ are $\geq 5$.

Let $X \supset S \supset C$ be a general surface hyperplane section and a general curve linear section of $X$. It follows that $C \subset Q_{3}^{0}$ where $Q_{3}^{0}$ is a smooth quadric in $\mathbb{P}^{4}$.

Since $C \subset Q_{3}^{0}$ is subcanonical, by the Serre construction, it is the zero locus of a section of a rank 2 bundle $\mathcal{E}$ on $Q_{3}^{0}$. If we now denote by $\zeta_{1}$ the hyperplane section and by $\zeta_{2}$ the class of a line on $Q_{3}^{0}$, we infer $c_{1}(\mathcal{E})=5 \zeta_{1}$ and $c_{2}(\mathcal{E})=13 \zeta_{2}$. Thus, for $\mathcal{F}=\mathcal{E}(-3)$ we compute $c_{1}(\mathcal{F})=-\zeta_{1}$ and $c_{2}(\mathcal{F})=\zeta_{2}$. We could not find in the literature a proof of the following:

Claim Let $\mathcal{F}$ be a rank two vector bundle on a smooth three- dimensional quadric with $c_{1}(\mathcal{F})=-1$ and $c_{2}(\mathcal{F})=1$. Then $\mathcal{F}$ is aCM, i.e., $h^{1}(\mathcal{F}(n))=0$ for all $n \in \mathbb{Z}$.

Indeed, from [2, p. 205], we know that the spinor bundle is the only stable bundle with these invariants and the spinor bundle is arithmetically Cohen-Macaulay. Hence, the claim is proved for stable $\mathcal{F}$.

Suppose now that $\mathcal{F}$ is not stable, i.e., we have $h^{0}(\mathcal{F}) \neq 0$. Clearly, $\mathcal{F}$ cannot be decomposable since a generic section of $\mathcal{F}(3)=\mathcal{E}$ defines a codimension 2 curve of odd degree. This means that a general section of $\mathcal{F}$ vanishes in codimension 2 along a curve of degree $c_{2}(\mathcal{F})=1$. Let us denote this line by $l$. From the exact sequences

$$
\begin{aligned}
& 0 \rightarrow \mathcal{O}_{Q_{3}^{0}}(n) \rightarrow \mathcal{F}(n) \rightarrow \mathcal{I}_{l \mid Q_{3}^{0}}(n-1) \rightarrow 0 \\
& 0 \rightarrow \mathcal{O}_{\mathbb{P}^{4}}(n-2) \rightarrow \mathcal{I}_{l}(n) \rightarrow \mathcal{I}_{l \mid Q_{3}^{0}}(n) \rightarrow 0
\end{aligned}
$$

we deduce that $h^{1}(\mathcal{F}(n))=h^{1}\left(\mathcal{I}_{l \mid Q_{3}^{0}}(n-1)\right)=h^{1}\left(\mathcal{I}_{l}(n-1)\right)$ for all $n \in \mathbb{Z}$. Since the line $l$ is aCM, we have $h^{1}(\mathcal{F}(n))=0$ for all $n \in \mathbb{Z}$. This proves the claim.

Applying the claim to the cohomology of the exact sequence

$$
0 \rightarrow \mathcal{O}_{Q_{3}^{0}}(n) \rightarrow \mathcal{F}(n+3) \rightarrow \mathcal{I}_{C \mid Q_{3}^{0}}(n+5) \rightarrow 0,
$$

it follows that $h^{1}\left(\mathcal{I}_{C \mid Q_{3}^{0}}\right)(n)=0$ for all $n \in \mathbb{Z}$. Now, from the exact sequence

$$
0 \rightarrow \mathcal{O}_{\mathbb{P}^{4}}(n-2) \rightarrow \mathcal{I}_{C}(n) \rightarrow \mathcal{I}_{C \mid Q_{3}^{0}}(n) \rightarrow 0,
$$

we infer that $h^{1}\left(\mathcal{I}_{C}(n)\right)=0$ for all $n \in \mathbb{Z}$.

Consider now the following exact sequences:

$$
\begin{aligned}
& 0 \rightarrow \mathcal{I}_{X}(n-1) \rightarrow \mathcal{I}_{X}(n) \rightarrow \mathcal{I}_{S}(n) \rightarrow 0 \\
& 0 \rightarrow \mathcal{I}_{S}(n-1) \rightarrow \mathcal{I}_{S}(n) \rightarrow \mathcal{I}_{C}(n) \rightarrow 0 .
\end{aligned}
$$

We know that $h^{2}\left(\mathcal{I}_{X}(n)\right)=0$ and, from Proposition 2.1, that $h^{1}\left(\mathcal{I}_{X}(1)\right)=0$. From the long exact sequences of cohomology corresponding to 5.1, we deduce that $h^{1}\left(\mathcal{I}_{S}(1)\right)=0$. Since $h^{1}\left(\mathcal{I}_{C}(n)\right)=0$ for all $n \in \mathbb{Z}$, it follows by induction that the long exact sequence constructed from (5.2) implies $h^{1}\left(\mathcal{I}_{S}(n)\right)=0$. Then, by the sequence (5.1), we have $h^{1}\left(\mathcal{I}_{X}(n)\right)=0$, so $X \subset \mathbb{P}^{6}$ is aCM. The assertion follows from Corollary 3.7.

To complete the classification of Calabi-Yau threefolds contained in quadrics, we lack only the consideration of Calabi-Yau threefolds of degree 14. For this, let us analyze more precisely Calabi-Yau threefolds contained in smooth 5-dimensional quadrics. Let $X \subset Q_{5}^{0}$ 
be a Calabi-Yau threefold. Since $X$ is subcanonical, from the Serre construction, we obtain that $X$ is the zero locus of a section of a rank 2 vector bundle $\mathcal{E}$, i.e.,

$$
0 \rightarrow \mathcal{O}_{Q_{5}^{0}} \rightarrow \mathcal{E} \rightarrow \mathcal{I}_{X \mid Q_{5}^{0}}\left(c_{1}(\mathcal{E})\right) \rightarrow 0 .
$$

Since $K_{X}=0$ we obtain $c_{1}(\mathcal{E})=5 \zeta_{1}$ where $\zeta_{1} \subset Q_{5}^{0}$ is the hyperplane section of $Q_{5}^{0}$. Moreover, we have $c_{2}(\mathcal{E})=\frac{1}{2} \operatorname{deg}(X) \zeta_{2}$, where $\zeta_{2}$ is a general codimension two linear section of $Q_{5}^{0}$. Finally $H^{i}(\mathcal{E}(k))=H^{i}\left(\mathcal{I}_{X \mid Q_{5}^{0}}\left(c_{1}(\mathcal{E})+(5+k)\right)\right.$.

It follows, by applying the Beilinson type spectral sequence as in [32], that the only arithmetically Cohen-Macaulay bundles (i.e, with vanishing intermediate cohomology) on $Q_{n}^{0}$ with $5 \geq n \geq 3$ are direct sums of line bundles and spinor bundles (see also [26]). Moreover, in [5, Theorem 1.7], it is proved that arithmetically Buchsbaum rank 2 vector bundles on $Q_{5}^{0}$, that are not aCM, are twists of Cayley bundles. Recall that a Cayley bundle $\mathcal{C}$ is defined in [30] by the exact sequences

$$
\begin{aligned}
& 0 \rightarrow \mathcal{O}_{Q_{5}^{0}} \rightarrow \mathcal{S} \rightarrow G \rightarrow 0, \\
& 0 \rightarrow \mathcal{O}_{Q_{5}^{0}} \rightarrow G(1) \rightarrow \mathcal{C}(1) \rightarrow 0,
\end{aligned}
$$

where $G$ is obtained by choosing a general section of the spinor bundle $\mathcal{S}$. It means that Calabi-Yau threefolds given as zero loci of sections of arithmetically Buchsbaum bundles on $Q_{5}^{0}$ are either degree 12 complete intersections or zero loci of twists of Cayley bundles. We saw in Corollary 5.1 that in degree 12, the complete intersections form a unique family of examples. We prove a similar result in the case of degree 14. Let us first construct two families of Calabi-Yau threefolds of degree 14.

Proposition 5.2 The zero locus of a generic section of the homogenous bundle $\mathcal{C}(3)$ is a Calabi-Yau threefold of degree 14 contained in $Q_{5}^{0}$.

Proof We have $c_{1}(\mathcal{C}(k))=2 k-1$ thus to obtain a Calabi-Yau threefold we put $k=3$. Then $c_{2}(\mathcal{C}(3))=14$. We know from [30, Theorem 3.7] that $\mathcal{C}(2)$ is globally generated. We deduce that the zero locus of a general section $\mathcal{C}(3)$ is a smooth Calabi-Yau threefold of degree 14.

The following family of Calabi-Yau threefolds of degree 14 was introduced in [7].

Proposition 5.3 Let $E_{14}=\Omega_{\mathbb{p 6}}^{1}(1) \oplus \mathcal{O}_{\mathbb{P}^{6}}(1)$. Then the degeneracy locus $D_{6}(\phi)$ of a generic skew-symmetric map $\phi: E_{14}^{*}(-1) \rightarrow E_{14}$ is a Calabi-Yau threefold of degree 14.

Proof Let $B_{14}$ be a general variety obtained by this construction. By the Bertini type theorem for Pfaffian subvarieties given in [29, Sect. 3] to prove that $B_{14}$ is a smooth threefold, it is enough to prove that the bundle $\bigwedge^{2} E_{14}(1)$ is globally generated. Now, $\bigwedge^{2} E_{14}(1)=$ $\Omega_{\mathbb{P}^{6}}^{2}(3) \oplus \Omega_{\mathbb{P}^{6}}^{1}(3)$. The Euler sequence gives us:

$$
0 \rightarrow \Omega_{\mathbb{P}^{6}}^{1}(1) \rightarrow 7 \mathcal{O}_{\mathbb{P} 6} \rightarrow \mathcal{O}_{\mathbb{P}^{6}}(1) \rightarrow 0 .
$$

Taking its second and third wedge powers we have:

$$
0 \rightarrow \Omega_{\mathbb{P}^{6}}^{2}(2) \rightarrow\left(\begin{array}{l}
7 \\
2
\end{array}\right) \mathcal{O}_{\mathbb{P}^{6}} \rightarrow \Omega^{1}(2) \rightarrow 0
$$

and

$$
0 \rightarrow \Omega_{\mathbb{P}^{6}}^{3}(3) \rightarrow\left(\begin{array}{l}
7 \\
3
\end{array}\right) \mathcal{O}_{\mathbb{P}^{6}} \rightarrow \Omega_{\mathbb{P}^{6}}^{2}(3) \rightarrow 0
$$


It follows that $\Omega_{\mathbb{P}^{6}}^{1}(2)$ and $\Omega_{\mathbb{P}^{6}}^{2}(3)$ are globally generated, hence, $\Omega_{\mathbb{P}^{6}}^{1}(3)$ and $\Omega_{\mathbb{P}^{6}}^{2}(3) \oplus \Omega_{\mathbb{P}^{6}}^{1}(3)$ are also globally generated. It follows that $B_{14}$ is a Pfaffian variety associated with the bundle $E_{14}=\Omega_{\mathbb{P} 6}^{1}(1) \oplus \mathcal{O}_{\mathbb{P}^{6}}(1)$, with $t=1$. The vanishing of the dualizing sheaf is then given by the adjunction formula (1.2) for Pfaffian varieties and the vanishing of the cohomology by the Pfaffian sequence 1.1.

We shall denote by $\mathfrak{C}_{14}$ and $\mathfrak{B}_{14}$ the families of Calabi-Yau threefolds obtained in Proposition 5.2 and Proposition 5.3, respectively. Observe that a generic $B_{14} \in \mathfrak{B}_{14}$ is contained in a quadric. Indeed, among the equations defining $B_{14}$, we have a section of the bundle $\left(\bigwedge^{6}\left(\Omega_{\mathbb{P} 6}^{1}(1)\right)\right)(3)=\mathcal{O}_{\mathbb{P}^{6}}(2)$ corresponding to the Pfaffian of a map $\left(\Omega_{\mathbb{P} 6}^{1}(1)\right)^{*}(-1) \rightarrow \Omega_{\mathbb{P} 6}^{1}(1)$.

We can now classify all Calabi-Yau threefolds of degree 14 contained in a 5-dimensional quadric.

Corollary 5.4 If $X$ is a Calabi-Yau threefold of degree 14 contained in a smooth quadric $Q_{5}^{0}$, then $X$ is obtained as the zero locus of a section of the twisted Cayley bundle $\mathcal{C}(3)$. Moreover, if $X$ is a Calabi-Yau threefold contained in any quadric, it is given by the Pfaffian construction applied to $E_{14}=\Omega_{\mathbb{P} 6}^{1}(1) \oplus \mathcal{O}_{\mathbb{P} 6}(1)$.

Proof Let $X$ be a Calabi-Yau threefold of degree 14 contained in the smooth quadric $Q_{5}^{0}$. By the Serre construction, we know that $X$ is given as the zero locus of a section of a rank 2 vector bundle $\mathcal{E}$ such that $c_{1}(\mathcal{E}(-3))=-\zeta_{1}$ and $c_{2}(\mathcal{E}(-3))=\zeta_{2}$. It follows from the main theorem in [30] that $\mathcal{E}(-3)$ is a Cayley bundle if we assume it is stable. To prove that $\mathcal{E}(-3)$ is stable is the same as to prove that $h^{0}(\mathcal{E}(-3))=h^{0}\left(\mathcal{I}_{X \mid Q_{5}^{0}}(2)\right)=0$ [see (5.3)]. To prove that $X$ is not contained in a second quadric (i.e., that $h^{0}\left(\mathcal{I}_{X \mid Q_{5}^{0}}(2)\right)=0$ ), we argue as in the proof of Corollary 5.1. More precisely, if $\mathcal{E}(-3)$ is not stable, then its general section defines a degree 2 threefold $W$ contained in $Q_{5}^{0}$. Since $Q_{5}^{0}$ contains no 3-dimensional linear space, $W$ must be a 3 -dimensional quadric. Hence, $W$ is aCM implying $\mathcal{E}$ is aCM and in consequence $X$ is aCM. Thus, $X$ is not contained in any quadric and this gives a contradiction.

To prove the second part, we first show that $h^{1}\left(\mathcal{I}_{X}(k)\right)=0$ for $k \neq 2$ and $h^{1}\left(\mathcal{I}_{X}(2)\right)=1$. From the long cohomology exact sequence obtained from 5.1 and 5.2, we infer $h^{1}\left(\mathcal{I}_{X}(n)\right)=$ $h^{1}\left(\mathcal{I}_{Z}(n)\right)$ where $C \subset \mathbb{P}^{4}$ is a codimension 2 linear section of $X \subset \mathbb{P}^{6}$. But we know that $X$ is contained in a quadric of rank $\geq 5$ thus $C$ is contained in a smooth quadric. It follows that $C$ is given as the zero locus of a section of a rank 2 vector bundle $\mathcal{B}(3)$ such that $c_{1}(\mathcal{B})=-\zeta_{1}$ and $c_{2}(\mathcal{B})=\zeta_{2}$. It follows that the zero locus of a general section of $\mathcal{B}$ is a sum $t$ of two skew lines. We find that the cohomologies of $h^{1}\left(\mathcal{I}_{t \mid \mathbb{P}^{3}}(k)\right)$ are 0 for $k \neq 2$ and 1 for $k=2$. Since all nonzero elements of the Hartshorne-Rao module of $X$ are of the same weight, it follows that $X$ is quasi-Buchsbaum. Hence, we deduce from Theorem 3.2 that $X=\operatorname{Pf}(\sigma)$ for some $\sigma \in H^{0}\left(\bigwedge^{2} E_{14}(1)\right)$ where $E_{14}=\Omega_{\mathbb{P}^{6}}^{1}(1) \oplus \mathcal{O}_{\mathbb{P}^{6}}(1)$. We thus get the assertion.

As a direct consequence of the above, we have the following.

Corollary 5.5 The family $\mathfrak{C}_{14}$ is contained in $\mathfrak{B}_{14}$ as a dense subset.

\section{Classification of degree 14 Calabi-Yau threefolds in $\mathbb{P}^{6}$}

The aim of this section is the classification of all degree 14 Calabi-Yau threefolds in $\mathbb{P}^{6}$.

From the Riemann-Roch theorem, we deduce that if $X \subset \mathbb{P}^{6}$ is a Calabi-Yau threefold of degree 14 then $h^{1}\left(\mathcal{I}_{X}(2)\right)=h^{0}\left(\mathcal{I}_{X}(2)\right)$ and $h^{1}\left(\mathcal{I}_{X}(3)\right)+7=h^{0}\left(\mathcal{I}_{X}(3)\right)$ (recall that $\left.h^{0}\left(\mathcal{I}_{X}(1)\right)=0\right)$. We have two possibilities: 
- $X$ is contained in a quadric,

- $X$ is not contained in any quadric.

The first case is solved by Corollary 5.4. Assume, hence, that $X$ is not contained in any quadric. Then there is an at least 7-dimensional space of cubics vanishing along $X$. We, moreover, claim the following.

Proposition 6.1 The singular locus of a generic cubic from $H^{0}\left(\mathcal{I}_{X}(3)\right)$ has dimension $\leq 1$.

Before we pass to the proof of Proposition 6.1, let us prove the following.

Lemma 6.2 Suppose that a Calabi-Yau threefold in $\mathbb{P}^{6}$ is not contained in any quadric. Let $X \supset S \supset C \supset F$ be general linear sections of $X$ of codimension 1,2,3, respectively. Then, we have $h^{0}\left(\mathcal{I}_{X}(3)\right)=h^{0}\left(\mathcal{I}_{S}(3)\right)=h^{0}\left(\mathcal{I}_{C}(3)\right)=h^{0}\left(\mathcal{I}_{F}(3)\right)$ and $0=h^{0}\left(\mathcal{I}_{X}(2)\right)=$ $h^{0}\left(\mathcal{I}_{S}(2)\right)=h^{0}\left(\mathcal{I}_{C}(2)\right)=h^{0}\left(\mathcal{I}_{F}(2)\right)$.

Proof First, we have $h^{i}\left(\mathcal{I}_{X}(k)\right)=0$ for $i=2,3$ and $k \in \mathbb{Z}$. Furthermore, we assumed $0=h^{0}\left(\mathcal{I}_{X}(m)\right)=h^{1}\left(\mathcal{I}_{X}(m)\right)$ for $m=1,2$. From the exact sequence

$$
0 \rightarrow \mathcal{I}_{X} \rightarrow \mathcal{I}_{X}(1) \rightarrow \mathcal{I}_{S}(1) \rightarrow 0
$$

tensorized by line bundles, we infer:

- $h^{i}\left(\mathcal{I}_{S}(m)\right)=0$ for $i=0,1,2$ and $m=0,1$;

- $h^{i}\left(\mathcal{I}_{S}(2)\right)=0$ for $i=1,2$;

- $H^{0}\left(\mathcal{I}_{X}(3)\right) \simeq H^{0}\left(\mathcal{I}_{S}(3)\right)$.

We conclude by repeating this procedure for a hyperplane section and a codimension 2 linear section.

We can now pass to the proof of Proposition 6.1

Proof of Proposition 6.1 We keep the notation from Lemma 6.2. By Lemma 6.2, it is enough to prove that the general cubic hypersurface in $\mathbb{P}^{4}$ containing $C$ is smooth. For this, we need to study the scheme-theoretic intersection of all cubics containing $C$ or more generally the scheme-theoretic intersection of cubics containing $X$. Let $\Lambda$ be the scheme-theoretic intersection of the cubics containing $X$. Then either $X$ is a component of $\Lambda$ (necessarily of multiplicity 1 for degree reason), or $\Lambda$ has a component $Y \subset \mathbb{P}^{6}$ of codimension 2 such that $X \subset Y$.

In the first case, we use the excess intersection formula. We choose $C_{1}, \ldots, C_{6}$ generic cubics containing $X$. Then we find $\left(C_{1} \ldots C_{6}\right)^{X}$ the equivalences of $X$ in the intersection $C_{1} \ldots C_{6}$. Indeed, from [19, prop. 9.1.1], we infer

$$
\left(C_{1} \ldots C_{6}\right)^{X}=\left(\sum_{i=1}^{6} c\left(\left.N_{C_{i} \mid \mathbb{P}^{6}}\right|_{X}\right) c\left(\left.T_{\mathbb{P}^{6}}\right|_{X}\right) c\left(T_{X}\right)\right)_{0}=c_{3}+15 h^{3}-11 h \cdot c_{2}=3^{6}-1
$$

that is an element from $A_{0}(X)$. The equivalences of all the distinguished components of $C_{1} \cap$ $\ldots \cap C_{6}$ sum up to $C_{1} \ldots C_{6}$ (see [19, \& 6.1] for an introduction to this method). Moreover, by the refined Bezout theorem [19, Theorem 12.3], the equivalences of the distinguished components are positive numbers of degree bigger than the degree of this component and all the irreducible components of $\bigcap C_{i}$ are among the distinguished components. It follows that there can be at most one such component whose support is not contained in $X$ and this component has degree 1 with multiplicity 1 in the intersection of $C_{i}$ for $1 \leq i \leq 6$. Since the 
equivalences of linear spaces of dimension $\geq 1$ in the intersection of cubics are bigger than 2, we deduce that there can be only one point outside $X$ in the intersection $\bigcap_{i=1}^{6} C_{i}$.

Let us now consider in this case a general codimension 2 linear section $C$ of $X \subset \mathbb{P}^{6}$. We can choose it not to pass through the additional point. We then have that $C$ is set theoretically defined by the cubics containing it. Moreover, $C$ is a component of multiplicity one of the scheme-theoretic intersections of these cubics. It follows that $C$ is scheme theoretically defined by the cubics containing it outside possibly a finite set $P$ of point on $C$. Then we know from [13, Theorem 2.1] that the generic cubic containing $C$ is smooth outside the set $P$. Suppose that there is a point from $P$ such that the generic cubic containing $C$ is singular in it. Since the choice of the codimension 2 linear section giving $C$ was generic, it means that the intersection of the singular loci of all cubics containing $X$ contains a surface $U$. Then each cubic containing $X$ must contain the secant variety $\mathfrak{S}_{2}(U)$ of the surface $U$. As we already saw that set theoretically, the intersection defines $X$ plus possibly one point we infer $\mathfrak{S}_{2}(U) \subset X$. Since $X$ is a Calabi-Yau threefold, we have $\mathfrak{S}_{2}(U) \neq X$ and, hence, $\mathfrak{S}_{2}(U)$ must be a surface. It follows that $U$ is a plane contained in $X$. But then the fiber of the projection from $U \subset \mathbb{P}^{6} \rightarrow \mathbb{P}^{3}$ intersects the cubics containing $X$ in linear spaces outside $U$. Thus, either $X$ is rational or $X$ is covered by lines. This is a contradiction in any case.

Assume now that $\Lambda$ has a component $Y \subset \mathbb{P}^{6}$ of codimension 2 such that $X \subset Y$. We still keep the notation from Lemma 6.2. Observe that, in this case, all the cubics in $\mathbb{P}^{3}$ containing $F$ contain also a fixed curve $D \subset \mathbb{P}^{3}$ being the codimension 3 linear section of $Y$. Moreover, since $X \subset Y$, we have $F \subset D$. By Lemma 6.2, the projective linear system of cubics containing $D$ is of dimension at least 6 . Moreover, since $F$ is not contained in any quadric, the restrictions of these cubics to a general hyperplane in $\mathbb{P}^{3}$ form a projective linear system of cubics on $\mathbb{P}^{2}$ of the same dimension 6 . It follows that the intersection of $D$ with a generic hyperplane is a scheme of length at most 3 . Hence, $D$ is a curve of degree 3 in $\mathbb{P}^{3}$. Such a curve is either contained in a hyperplane or in a quadric hypersurface. The latter is a contradiction with $F \subset D$ and $h^{0}\left(\mathcal{I}_{F}(2)\right)=0$.

Corollary 6.3 A Calabi-Yau threefold of degree 14 in $\mathbb{P}^{6}$ that is not contained in any quadric is defined by the $6 \times 6$ Pfaffians of a skew $7 \times 7$ matrix with linear entries.

Proof Let us consider a generic codimension 2 linear section $C$ of $X \subset \mathbb{P}^{6}$. From Proposition 6.1 , the curve $C$ is contained in a smooth cubic threefold $W$. Let $H$ be the class of the hyperplane section of $W$. Since $K_{C}=2 H$ and $K_{T}=-2 H$, we deduce that $C \subset W$ is subcanonical. From the Kawamata-Viehweg vanishing theorem, we have $h^{1}\left(\mathcal{O}_{W}(-4 H)\right)=$ 0 and $h^{2}\left(\mathcal{O}_{T}(-8 H)\right)$. Thus, we can apply the Serre construction and find a rank 2 vector bundle $\mathcal{E}$ on $W$ with a section vanishing along $C \subset W$. More precisely, we obtain an exact sequence

$$
0 \rightarrow \mathcal{O}_{W} \rightarrow \mathcal{E} \rightarrow \mathcal{I}_{C \mid W}(4 H) \rightarrow 0
$$

and $c_{1}(\mathcal{E})=4 H$ and $H \cdot c_{2}(\mathcal{E})=14$. We compute that $c_{1}(\mathcal{E}(-2))=0$ and $H \cdot c_{2}(\mathcal{E}(-2))=$ 2. Moreover, $\mathcal{E}$ is stable if and only if $h^{0}(\mathcal{E}(-2))=0$. From the exact sequence $(6.1)$ tensorized by $\mathcal{O}(-2 H)$, we deduce that $h^{0}(\mathcal{E}(-2)) \neq 0$ implies that $h^{0}\left(\mathcal{I}_{C \mid W}(2)\right) \neq 0$. This contradicts the fact that $C$ is not contained in any quadric. Thus, $\mathcal{E}$ is stable. By Serre duality, $0=h^{2}(\mathcal{E}(-2))=h^{0}(\mathcal{E}(-3))$. It is proven in [14, Theorem 2.4] that if $\mathcal{E}(-2)$ is stable, then $h^{i}(\mathcal{E}(-1-i))=0$ for $i \geq 1$. It follows that $h^{1}(\mathcal{E}(n))=0$ for $n \in \mathbb{Z}$. Now, from the long exact sequence of cohomology associated with the exact sequence (6.1), we infer $h^{1}\left(\mathcal{I}_{C \mid W}(4-k)\right) \leq h^{2}\left(\mathcal{O}_{W}(-k)\right)=h^{1}\left(\mathcal{O}_{W}(-2+k)\right)$. From the Kodaira vanishing theorem, the last number is 0 so $\left.h^{1}\left(\mathcal{I}_{C \mid W}(k)\right)\right)=0$. Next, from the long exact sequence of cohomology associated with the sequence 


$$
0 \rightarrow \mathcal{O}_{\mathbb{P}^{4}}(-3) \rightarrow \mathcal{I}_{C \mid \mathbb{P}^{4}} \rightarrow \mathcal{I}_{C \mid W} \rightarrow 0
$$

we obtain

$$
\left.h^{1}\left(\mathcal{I}_{C \mid \mathbb{P}^{4}}(k)\right)\right)=0 .
$$

Finally, arguing as in Lemma 6.2 we infer $h^{1}\left(\mathcal{I}_{X}(k)\right)=0$ for $k \in \mathbb{Z}$ thus $X \subset \mathbb{P}^{6}$ is aCM. The assertion follows from Corollary 3.7.

\section{Classification up to deformations}

By the classification of Sect. 5 for $d \leq 13$, the Hilbert scheme of Calabi-Yau threefolds of degree $d$ has a unique irreducible component. In this section, we prove that the statement is also valid for $d=14$. To do this, we compare the families of Calabi-Yau threefolds of degree 14 in $\mathbb{P}^{6}$ appearing in the classification above. We show that all such varieties are smooth degenerations of the family of Calabi-Yau threefolds of degree 14 defined by the $6 \times 6$ Pfaffians of an alternating $7 \times 7$ matrix, and hence, they are in the same component of the Hilbert scheme.

Denote by $\mathfrak{T}_{14}$ the family of Calabi-Yau threefold defined by $6 \times 6$ Pfaffians of $7 \times 7$ matrices of linear forms. We observed that the general element $T_{14} \in \mathfrak{T}_{14}$ is not contained in any quadric. On the other hand, we have two families $\mathfrak{C}_{14} \subset \mathfrak{B}_{14}$ of Calabi-Yau threefolds contained in a quadric.

We compute the dimension of $\mathfrak{C}_{14}$ using Bott formula. From the computation and Corollary 5.5, it follows that the dimension of the component of the Hilbert scheme of Calabi-Yau threefolds of degree 14 in $\mathbb{P}^{6}$ containing $\mathfrak{B}_{14}$ is bigger than the dimension of $\mathfrak{B}_{14}$. In fact, we prove the following.

Theorem 7.1 For a generic Calabi-Yau threefold $B_{14}$ belonging to $\mathfrak{B}_{14}$, there exists a smooth morphism $\mathbb{P}^{6} \times \Delta \supset \mathcal{X} \rightarrow \Delta$ to the complex disk $\Delta$ such that for $\lambda \neq 0$ the fiber $\mathcal{X}_{\lambda}$ is a smooth subvariety in $\mathbb{P}^{6}$ belonging to the family $\mathfrak{T}_{14}$ whereas the central fiber $\mathcal{X}_{0}=B_{14}$.

The theorem is a straightforward consequence of the Euler sequence and the following Proposition 7.2.

To formulate the proposition let us start with two vector bundles $E, F$ of ranks $2 u$ and $2 u+1$ on $\mathbb{P}^{6}$ forming an exact sequence.

$$
0 \rightarrow E \rightarrow F \rightarrow \mathcal{O}_{\mathbb{P}^{6}}(1) \rightarrow 0
$$

Consider the exact sequence relating wedge squares of the bundles tensorized by $\mathcal{O}_{\mathbb{P}^{6}}(1)$ :

$$
0 \rightarrow\left(\bigwedge^{2} E\right)(1) \rightarrow\left(\bigwedge^{2} F\right)(1) \rightarrow E(2) \rightarrow 0
$$

and its associated cohomology sequence:

$$
0 \rightarrow H^{0}\left(\left(\bigwedge^{2} E\right)(1)\right) \stackrel{\eta}{\rightarrow} H^{0}\left(\left(\bigwedge^{2} F\right)(1)\right) \stackrel{\delta}{\rightarrow} H^{0}(E(2)) \rightarrow H^{1}\left(\left(\bigwedge^{2} E\right)(1)\right)
$$

Assume that the map $\delta$ is surjective. Assume moreover that the Pfaffian varieties $X_{\sigma}$ and $X_{\sigma^{\prime}}$ associated with generic sections $\sigma \in H^{0}\left(\left(\bigwedge^{2} E\right)(1) \oplus \mathcal{O}_{\mathbb{P} 6}(1)\right)$ and $\sigma^{\prime} \in H^{0}\left(\left(\bigwedge^{2} F\right)(1)\right)$ are irreducible of codimension 3 as expected. 
Proposition 7.2 Let $E, F$ be vector bundles as above. Then for a generic section $s \in$ $H^{0}\left(\left(\bigwedge^{2}\left(E \oplus \mathcal{O}_{\mathbb{P}^{6}}(1)\right)\right)(1)\right)$, there exists a family of sections $\sigma_{\lambda}^{\prime} \in H^{0}\left(\left(\bigwedge^{2} F\right)(1)\right)$ parametrized by $\lambda \in \mathbb{C} \backslash\{0\}$ such that the family

$$
X_{\lambda}=\left\{\begin{array}{l}
\operatorname{Pf}\left(\sigma_{\lambda}^{\prime}\right) \text { for } \lambda \neq 0 \\
\operatorname{Pf}(\sigma) \text { for } \lambda=0
\end{array}\right.
$$

is a flat family of 3-dimensional subvarieties of $\mathbb{P}^{6}$.

Proof Let $\sigma \in H^{0}\left(\left(\bigwedge^{2}\left(E \oplus \mathcal{O}_{\mathbb{P} 6}(1)\right)\right)(1)\right)$. We have $\sigma=\left(\sigma_{1}, \sigma_{2}\right)$ with $\left.\sigma_{1} \in H^{0}\left(\bigwedge^{2} E\right)(1)\right)$ and $\sigma_{2} \in H^{0}(E(2))$. By the assumption made, there exists a pair $\left(\sigma_{1}^{\prime}, \sigma_{2}^{\prime}\right)$ of sections $\sigma_{1}, \sigma_{2} \in$ $H^{0}\left(\bigwedge^{2} F\right)(1)$ such that $\eta\left(\sigma_{1}\right)=\sigma_{1}^{\prime}, \delta\left(\sigma_{2}^{\prime}\right)=\sigma_{2}$. Let $\sigma_{\lambda}^{\prime}=\sigma_{1}^{\prime}+\lambda \sigma_{2}^{\prime} \in H^{0}\left(\left(\bigwedge^{2} F\right)(1)\right)$.

Consider the variety $\Delta \times \mathbb{P}^{6}$ with projection $\pi_{\Delta}$ and $\pi_{\mathbb{P} 6}$. Consider now the subscheme $\tilde{\mathcal{X}} \subset \Delta \times \mathbb{P}^{6}$ defined by the vanishing of the section $\Delta \times \mathbb{P}^{6} \ni(\lambda, x) \mapsto\left((\lambda, x), \sigma_{\lambda}^{\prime}(x)^{(\wedge r)}\right) \in$ $\pi_{\mathbb{P} 6}^{*}\left(\left(\bigwedge^{2} u F\right)(u)\right)$, and consider $\mathcal{X}$ its irreducible component dominating $\Delta$. By [22, Proposition 9.7], the family $\pi_{\Delta}: \mathcal{X} \rightarrow \Delta$ is flat. We clearly see that the fiber $\mathcal{X}_{\lambda}$ for $\lambda \neq 0$ is $X_{\lambda}$. It is, hence, enough to prove that the fiber $\mathcal{X}_{0}$ over $0 \in \Delta$ is equal to $X_{0}$

We have $\left(\sigma_{1}^{\prime}+\lambda \sigma_{2}^{\prime}\right)^{\wedge u}=\left(\sigma_{1}^{\prime}\right)^{\wedge u}+\lambda \gamma_{1}$ for some $\gamma_{1} \in H^{0}\left(\left(\bigwedge^{2 u} F\right)(u)\right)$. It follows that $\mathcal{X}_{0}$ is contained in the zero locus $Z\left(\left(\sigma_{1}^{\prime}\right)^{\wedge u}\right)$ of the section $\left(\sigma_{1}^{\prime}\right)^{\wedge u} \in H^{0}\left(\bigwedge^{2 u} F\right)$. Moreover, by the exact sequence:

$$
0 \rightarrow\left(\bigwedge^{2 u} E\right)(u) \rightarrow\left(\bigwedge^{2 u} F\right)(u) \stackrel{\psi}{\rightarrow}\left(\bigwedge^{2 u-1} E\right)(u+1) \rightarrow 0
$$

we infer $Z\left(\left(\sigma_{1}^{\prime}\right)^{\wedge u}\right)=Z\left(\sigma_{1}^{\wedge u}\right)$. Furthermore, knowing that

$$
\psi\left(\left(\sigma_{1}^{\prime}+\lambda \sigma_{2}^{\prime}\right)^{\wedge u}\right)=\lambda \sigma_{1}^{\wedge u-1} \wedge \sigma_{2}+\lambda^{2} \gamma_{2}
$$

for some $\gamma_{2} \in H^{0}\left(\left(\bigwedge^{2 u} F\right)(u)\right)$ and that

$$
Z\left(\left(\sigma_{1}^{\prime}+\lambda \sigma_{2}^{\prime}\right)^{\wedge u}\right) \subset Z\left(\psi\left(\left(\sigma_{1}^{\prime}+\lambda \sigma_{2}^{\prime}\right)^{\wedge u}\right)\right),
$$

we see that $\mathcal{X}_{0} \subset Z\left(\sigma_{1}^{\wedge u-1} \wedge \sigma_{2}\right)$. Putting everything together, we get: $\mathcal{X}_{0}$ is contained in the zero locus of

$$
\begin{aligned}
\left(\sigma_{1}^{\wedge u}, \sigma_{1}^{\wedge u-1} \wedge \sigma_{2}\right) \in H^{0} & \left(\left(\bigwedge^{2 u}\left(E \oplus \mathcal{O}_{\mathbb{P}^{6}}(1)\right)\right)(u)\right) \\
= & H^{0}\left(\left(\bigwedge^{2} u E\right)(u)\right) \oplus H^{0}\left(\bigwedge^{2 u-1} E(u+1)\right)
\end{aligned}
$$

The latter zero locus is precisely the variety $X_{0}=\operatorname{Pf}\left(\left(\sigma_{1}, \sigma_{2}\right)\right)$. It follows that $\mathcal{X}_{0} \subset X_{0}$. But, since $\pi_{\Delta} \mid \mathcal{X}: \mathcal{X} \rightarrow \Delta$ is flat, we know that $\mathcal{X}_{0}$ is of codimension 3 in $\mathbb{P}^{6}$ which implies by assumption on $X_{0}$ that $\mathcal{X}_{0}=X_{0}$.

Proof of Theorem 7.1 The Euler sequence gives:

$$
0 \rightarrow \Omega_{\mathbb{P} 6}^{1}(1) \rightarrow 7 \mathcal{O}_{\mathbb{P} 6} \rightarrow \mathcal{O}_{\mathbb{P} 6}(1) \rightarrow 0 .
$$

By Proposition 7.2, for any $B_{14} \in \mathfrak{B}_{14}$, we obtain a flat family of manifolds defined as Pfaffians associated with the bundle $7 \mathcal{O}$ degenerating to $B_{14}$. Since smoothness is an open condition in flat families, we conclude that any smooth $B_{14}$ is a smooth degeneration of a family of smooth Calabi-Yau threefolds from $\mathfrak{T}_{14}$. 
Remark 7.3 Similarly, we prove using Proposition 7.2 that any Calabi-Yau threefold $B_{15}$ from Proposition 4.11 is a flat degeneration of the family of Calabi-Yau threefolds of degree 15 defined as Pfaffians of the bundle $\Omega_{\mathbb{P} 6}^{1}(1) \oplus 3 \mathcal{O}_{\mathbb{P} 6}$.

Acknowledgments We would like to thank C. Okonek for initiating the project, motivation, and help. We would like to thank L. Gruson and C. Peskine for mathematical inspiration. We would also like to thank A. Bertin, J. Buczynski, S. Cynk, D. Faenzi, P. Pragacz for helpful comments and the referee for helpful remarks and suggestions. The first author was supported by MNSiW, N N201 414539, and the second by the Forschungskredit of the University of Zurich and Iuventus Nr IP2011 005071 "Uklady linii na zespolonych rozmaitosciach kontaktowych oraz uogolnienia."

Open Access This article is distributed under the terms of the Creative Commons Attribution License which permits any use, distribution, and reproduction in any medium, provided the original author(s) and the source are credited.

\section{References}

1. Arrondo, E.: A home-made Hartshorne-Serre correspondence. Rev. Mat. Complut. 20(2), 423-443 (2007)

2. Arrondo, E., Sols, I.: Classification of smooth congruences of low degree. J. Reine Angew. Math. 393, 199-219 (1989)

3. Aure, A.B., Ranestad, K.: The smooth surfaces of degree 9 in $\mathbb{P}^{4}$. Complex projective geometry. London mathematical society lecture note series 179, pp. 32-46. Cambridge University Press, Cambridge (1992)

4. Ballico, E., Chiantini, L.: On smooth subcanonical varieties of codimension 2 in $\mathbb{P}^{n}, n \geq 4$. Ann. Math. Pura Appl. 135, 99-117 (1983)

5. Ballico, E., Malaspina, F., Valabrega, P., Valenzano, M.: On Buchsbaum bundles on quadric hypersurfaces. arXiv:1108.0075 [math.AG]

6. Beltrametti, M., Schneider, M., Sommese, A.J.: Threefolds of degree 9 and 10 in P5. Math. Ann. 288(3), 413-444 (1990)

7. Bertin, M.A.: Examples of Calabi-Yau 3-folds of $\mathbb{P}^{7}$ with $\rho=1$. Can. J. Math. 61(5), 1050-1072 (2009)

8. Böhm, J.: Mirror symmetry and tropical geometry. Ph.D. Thesis Universitat des Saarlandes (2008). arXiv:0708.4402v1 [math.AG]

9. Braun, R., Ottaviani, G., Schneider, M., Schreyer, F.O.: Boundedness for nongeneral-type 3-folds in P5. In: Complex Analysis and Geometry, pp. 311-338. Univ. Ser. Math., Plenum, New York (1993)

10. Catanese, F.: Homological algebra and algebraic surfaces. In: Algebraic Geometry, Santa Cruz 1995, pp. 3-56, Proceedings of Symposia in Pure Mathematics, 62, Part 1, American Mathematical Society, Providence, RI (1997)

11. Decker, W., Ein, L., Schreyer, F.O.: Construction of surfaces in P4. J. Algebr. Geom. 2, 185-237 (1993)

12. Decker, W., Popescu, S.: On surfaces in P4 and 3-folds in P5. In: Vector Bundles in Algebraic Geometry (Durham, 1993). London Math. Soc. Lecture Note Ser., vol. 208, pp. 69-100. Cambridge University Press, Cambridge (1995)

13. Diaz, S., Harbater, D.: Strong Bertini theorems. Trans. Am. Math. Soc. 324(1), 73-86 (1991)

14. Druel, S.: Espace des modules des faisceaux de rang 2 semi-stables de classes de Chern $c_{1}=0, c_{2}=2$ et $c_{3}=0$ sur la cubique de $\mathbb{P}^{4}$. Int. Math. Res. Notices 2000(19), 985-1004 (2000)

15. Ellingsrud, G., Peskine, C.: Sur les surfaces lisses de P4. Invent. Math. 95(1), 1-11 (1989)

16. Eisenbud, D., Popescu, S., Walter, C.: Lagrangian subbundles and codimension 3 subcanonical subschemes. Duke Math. J. 107(3), 427-467 (2001)

17. Ellia, P., Franco, D., Gruson, L.: Smooth divisors of projective hypersurfaces. Comment. Math. Helv. 83(2), 371-385 (2008)

18. Fujita, T.: Projective threefolds with small secant varieties. Sci. Pap. Coll. Gen. Educ. Univ. Tokyo 32(1), 33-46 (1982)

19. Fulton, W.: Intersection theory, 2nd edn. Ergebnisse der Mathematik und ihrer Grenzgebiete. 3. Folge. In: A Series of Modern Surveys in Mathematics. Springer, Berlin (1998). xiv+470 pp

20. Grayson, D.R., Stillman, M.E.: Macaulay2, a software system for research in algebraic geometry. http:// www.math.uiuc.edu/Macaulay2/

21. Hartshorne, R.: Genre de courbes algébriques dans l'espace projectif (d'après L. Gruson et C. Peskine). (French) [Genus of algebraic curves in projective space (after L. Gruson and C. Peskine)] Bourbaki Seminar, vol. 1981/1982, pp. 30117313, Astérisque, 92-1793, Soc. Math. France, Paris (1982) 
22. Hartshorne, R.: Algebraic Geometry. Graduate Texts in Mathematics, vol. 52. Springer, New York (1977). xvi+496 pp. ISBN:0-387-90244-9

23. Kapustka, G.: Projections of del Pezzo surface and Calabi-Yau threefolds. arXiv:1010.3895 [math.AG]

24. Kapustka, G., Kapustka, M.: Del Pezzo surfaces in $\mathbb{P}^{5}$ and Calabi-Yau threefolds in $\mathbb{P}^{6}$. arXiv:1310.0774 [math.AG]

25. Kapustka, G., Kapustka, M.: A cascade of determinantal Calabi-Yau threefolds. Math. Nachr. 283(12), 1795-1809 (2010)

26. Knorrer, H.: Cohen-Macaulay modules on hypersurface singularities. I. Invent. Math. 88(1), 153C164 (1987)

27. Miyaoka, Y.: The Chern classes and Kodaira dimension of a minimal variety. Adv. Stud.Pure Math. 10, 449-476 (1987)

28. Oguiso, K., Sakurai, J.: Calabi-Yau threefolds of quotient type. Asian J. Math. 5(1), $43-77$ (2001)

29. Okonek, C.: Note on subvariety of codimension 3 in $\mathbb{P}^{n}$. Manuscr. Math. 84(3-4), 421-442 (1994)

30. Ottaviani, G.: On Cayley bundles on the five-dimensional quadric. Boll. Un. Mat. Ital. A (7) 4(1), 87-100 (1990)

31. Rødland, E.A.: The Pfaffian Calabi-Yau, its mirror, and their link to the Grassmannian $G(2,7)$. Compos. Math. 122(2), 135-149 (2000)

32. Sols, I.: On spinor bundles. J. Pure. Appl. Algebra 35, 85-94 (1985)

33. Schneider, M.: Boundedness of low-codimensional submanifolds of projective space. Int. J. Math. 3(3), 397-399 (1992)

34. Schreyer, F.-O., Tonoli, F.: Needles in a haystack: special varieties via small fields. In: Computations in Algebraic Geometry with Macaulay, vol. 2, pp. 251-279, Algorithms Comput. Math., 8. Springer, Berlin (2002)

35. Tonoli, F.: Construction of Calabi-Yau 3-folds in $\mathbb{P}^{6}$. J. Algebr. Geom. 13(2), 209-232 (2004)

36. Van de Ven, A.: On the embedding of abelian varieties in projective spaces. Ann. Mat. Pura Appl. (4) 103, 127-129 (1975)

37. Walter, C.H.: Pfaffian subscheme. J. Algebr. Geom. 5(4), 671-704 (1996)

38. Zak, F.: Tangents and Secants of Algebraic Varieties. Translations of Mathematical Monographs, vol. 127. American Mathematical Society, Providence, RI (1993). viii+164 pp 